\title{
NORMALIZED SOLUTIONS AND MASS CONCENTRATION FOR SUPERCRITICAL NONLINEAR SCHRÖDINGER EQUATIONS
}

\author{
JIANFU YANG \\ Department of Mathematics, Jiangxi Normal University \\ Nanchang, Jiangxi 330022, P. R. China \\ email: Jianfu Yang: jfyang_2000@yahoo.com \\ JINGE YANG* \\ School of Sciences, Nanchang Institute of Technology \\ Nanchang 330099, P. R. China \\ email: Jinge Yang: jgyang2007@yeah.net
}

\begin{abstract}
In this paper, we deal with the existence and concentration of normalized solutions to the supercritical nonlinear Schrödinger equation

$$
\left\{\begin{array}{l}
-\Delta u+V(x) u=\mu_{q} u+a|u|^{q} u \text { in } \mathbb{R}^{2}, \\
\int_{\mathbb{R}^{2}}|u|^{2} d x=1
\end{array}\right.
$$

where $\mu_{q}$ is the Lagrange multiplier. We show that for $q>2$ close to 2 , the equation admits two solutions: one is the local minimal solution $u_{q}$ and another one is the mountain pass solution $v_{q}$. Furthermore, we study the limiting behavior of $u_{q}$ and $v_{q}$ when $q \rightarrow 2_{+}$. Particularly, we describe precisely the blow-up formation of the excited state $v_{q}$.
\end{abstract}

\section{INTRODUCTION}

In this paper, we study the existence and asymptotic behavior of standing waves for the following nonlinear Schrödinger equation

$$
i \psi_{t}(x, t)=-\Delta \psi(x, t)+V(x) \psi(x, t)-a|\psi(x, t)|^{q} \psi(x, t) \quad(x, t) \in \mathbb{R}^{2} \times \mathbb{R}^{1},
$$

where $a>0, q>2$, and $V$ is an external potential. The wave function $\psi$ is confined to the mass constraint $\int_{\mathbb{R}^{2}}|\psi|^{2} d x=1$.

By a standing wave of (1.1) we mean a solution of equation (1.1) with the form $\psi(t, x)=$ $e^{i \omega t} u(x)$. In particular, the function $u$ satisfies

$$
-\Delta u+(V(x)+\omega) u=a|u|^{q} u \text { in } \mathbb{R}^{2}
$$

and

$$
\int_{\mathbb{R}^{2}}|u(x)|^{2} d x=1
$$

In the case $q=2$, equation (1.1) stems from the study of Bose-Einstein condensation. It was derived independently by Gross and Pitaevskii, and it is the main theoretical tool

Key words: $L^{2}$ supercritical, constrained problems, existence, asymptotic behavior. 
for investigating nonuniform dilute Bose gases at low temperatures. Especially, equation (1.2) is called the Gross-Pitaevskii equation. The constant $a$ is the interaction coupling constant fixed by the $s$-wave scattering length. The case $a>0$ represents that the force between the atoms in the condensates is attractive, and if $a<0$, the force is repulsive. Bose-Einstein condensates with attractive interactions in two dimensions, are described by the Gross-Pitaevskii (GP) energy functional

$$
E_{a}(u)=\frac{1}{2} \int_{\mathbb{R}^{2}}\left(|\nabla u(x)|^{2}+V(x)|u(x)|^{2}\right) d x-\frac{a}{q+2} \int_{\mathbb{R}^{2}}|u(x)|^{4} d x .
$$

The exponent 4 is critical for the functional $E_{a}(u)$ under the unit mass constraint (1.3) in the sense that if we make a transformation $u_{\lambda}(x)=\lambda \varphi(\lambda x), \lambda>0$ for any fixed $\varphi \in H^{1}\left(\mathbb{R}^{2}\right)$ with $\|\varphi\|_{L^{2}\left(\mathbb{R}^{2}\right)}=1$ in the energy functional

$$
E_{a, q}(u)=\frac{1}{2} \int_{\mathbb{R}^{2}}\left(|\nabla u(x)|^{2}+V(x)|u(x)|^{2}\right) d x-\frac{a}{q+2} \int_{\mathbb{R}^{2}}|u(x)|^{q+2} d x,
$$

then $\left\|u_{\lambda}\right\|_{L^{2}\left(\mathbb{R}^{2}\right)}=1$ and $E_{a, q}\left(u_{\lambda}\right)$ is bounded from below if $q<2$ and unbounded if $q>2$. We refer the cases $1<q<2, q=2$ and $q>2$ as $L^{2}$ subcritical, critical and supercritical respectively. Hence, the constrained minimization problem

$$
d_{a}(q):=\inf _{u \in \mathcal{H}, \int_{\mathbb{R}^{2}}|u|^{2} d x=1} E_{a, q}(u)
$$

can only be considered for subcritical and critical cases, where $\mathcal{H}$ is defined by

$$
\mathcal{H}:=\left\{u \in H^{1}\left(\mathbb{R}^{2}\right): \int_{\mathbb{R}^{2}} V(x)|u(x)|^{2} d x<\infty\right\} .
$$

If $q=2$, in the attractive case, the system of Bose-Einstein condensates collapses whenever the particle number increases beyond a critical value; see $[7,10,14,19]$ etc. Mathematically, it was proved in [11] that there exists a threshold value $a^{*}>0$ such that $d_{a}(2)$ is achieved if $0<a<a^{*}$, and there is no minimizer for $d_{a}(2)$ if $a \geq a^{*}$. The threshold value $a^{*}$ is determined in terms of the solution of the nonlinear scalar field equation

$$
-\Delta u+u=u^{3} \text { in } \mathbb{R}^{2}, \quad u \in H^{1}\left(\mathbb{R}^{2}\right) .
$$

It is known from [15] that problem (1.6) admits a unique positive solution up to translations. Such a solution is radially symmetric and exponentially decaying at infinity, see for instance, [5]. Denote by $Q$ in the sequel the positive solution of (1.6), which is radially symmetric about the origin. It was found in [11] that the threshold value $a^{*}$ is given by

$$
a^{*}:=\|Q\|_{L^{2}\left(\mathbb{R}^{2}\right)}^{2} .
$$

Furthermore, if $V$ is a trap potential, that is $V(x)=\prod_{i=1}^{n}\left|x-x_{i}\right|^{p}$ with $p>1$, it was shown in [11] that symmetry breaking occurs in the GP minimizers. For $a<a^{*}$ close to $a^{*}$, the GP functional $E_{a, 2}$ has at least $n$ different non-negative minimizers, each of which concentrates at a specific global minimum point $x_{i}$.

The similar symmetry breaking phenomenon was considered in the subcritical case, i.e. $0<q<2$, for the functional $E_{a, q}$ in [12]. When $q$ approaching 2 , the limit behavior of the 
minimizer of $E_{a, q}$ constrained by (1.3) is described by the unique positive solution $\varphi_{q}$ of the nonlinear scalar field equation

$$
-\Delta u+\frac{2}{q} u=\frac{2}{q} u^{q+1}, \quad q>0, \quad u \in H^{1}\left(\mathbb{R}^{2}\right) .
$$

In this paper, we consider the existence of solutions for the supercritical problem

$$
\left\{\begin{array}{l}
-\Delta u+V(x) u=\mu_{q} u+a|u|^{q} u \text { in } \mathbb{R}^{2}, \\
\int_{\mathbb{R}^{2}}|u|^{2} d x=1
\end{array}\right.
$$

as well as the asymptotic behavior of solutions. That is, we will study the case $q>2$. In the sequel, $\mu_{q}$ denotes the Lagrange multiplier.

Although in the supercritical case, there is no minimizer for the minimization problem (1.5), or no ground state solution for the problem

$$
-\Delta u+V(x) u=\mu_{q} u+a|u|^{q} u \text { in } \mathbb{R}^{2},
$$

we can find critical points of $E_{a, q}$ constrained on the manifold

$$
S(1)=\left\{u \in \mathcal{H}: \int_{\mathbb{R}^{2}}|u|^{2} d x=1\right\}
$$

Such a critical point is an excited state solution of (1.10). Actually, for the supercritical case, it was revealed in $[3,13]$ that the functional $E_{a, q}$ with $V=0$ has a mountain pass geometry on $S(1)$. Based on this observation, a variational method was developed to apply to various problems, see [2,4] etc. We will look for critical points of $E_{a, q}$ on $S(1)$. As observed, one critical point of $E_{a, q}$ on $S(1)$ can be found as a local minimizer, and another one can be obtained by a variant mountain pass theorem. In fact, we will show that the functional $E_{a, q}$ has a mountain pass geometry on $S(1)$, and it implies that there is a $(P S)$ sequence of $E_{a, q}$. In order to bound the (PS) sequence, inspired of [8] and [13] we establish a variant mountain pass theorem, in which the $(P S)$ sequence is found close to the Pohozaev manifold, see section 2 for details.

We assume that the potential function $V \in C^{1}\left(\mathbb{R}^{2}\right)$ satisfies

$\left(V_{1}\right): \lim _{|x| \rightarrow \infty} V(x)=\infty, \quad \inf _{x \in \mathbb{R}^{2}} V(x)=0$.

$\left(V_{2}\right):(q-1) V+x \cdot \nabla V \geq-C_{1}$ and $|x \cdot \nabla V(x)| \leq C_{2}(V(x)+1)$,

where $C_{i}>0, i=1,2$.

In the sequel, we choose $a \in\left(0, a^{*}\right)$. Denote $A_{k}=\left\{\left.u \in S(1)\left|\int_{\mathbb{R}^{2}}\right| \nabla u\right|^{2} d x \leq k\right\}$. The local minimizer will be found in $A_{k}$. In order to study the asymptotic behavior of critical points of $E_{a, q}$, the number $k$ needs to be selected carefully. Actually, we set

$$
\tau_{q}^{2}=\left(\frac{2 a_{q}^{*}}{q a}\right)^{\frac{2}{q-2}}
$$

where $a_{q}^{*}$ is defined later in (2.4). Then we obtain the following existence results.

Theorem 1.1. Suppose $V$ satisfies $\left(V_{1}\right)$ and $\left(V_{2}\right)$. There exists an $\varepsilon_{0}>0$ such that, for any $q \in\left(2,2+\varepsilon_{0}\right), E_{a, q}(u)$ admits a local positive minimizer $u_{q}$ in $A_{\tau_{q}^{2}}$, that is

$$
E_{a, q}\left(u_{q}\right)=\inf _{u \in A_{\tau_{q}^{2}}} E_{a, q}(u)
$$


and a second positive critical point $v_{q}$ at the mountain pass level on $S(1)$.

We may verify that the trap potential $V$, which has $n \geq 1$ isolated minima, and that in their vicinity $\mathrm{V}$ behaves like a power of the distance from these points, satisfies conditions $\left(V_{1}\right)$ and $\left(V_{2}\right)$. Precisely, we assume for $n \geq 1$ that

$\left(V_{e}\right) V(x)=\prod_{i=1}^{n}\left|x-x_{i}\right|^{p_{i}}$ with $p_{i} \geq 1, i=1, \cdots, n$.

Hence, we have in particular the following result.

Corollary 1.1. Suppose $V$ satisfies condition $\left(V_{e}\right)$. Then, $V$ satisfies $\left(V_{1}\right)$ and $\left(V_{2}\right)$. Consequently, the conclusions in Theorem 1.1 also hold.

Next, we study the asymptotic behavior of the local minimizer $u_{q}$ and the mountain pass point $v_{q}$ as $q \rightarrow 2_{+}$. For the supercritical case, it seems that no works concerning the asymptotic behavior of solutions can be found in the literature. In this paper, we give a precise description of the asymptotic behavior of solutions $u_{q}$ and $v_{q}$. We commence with the following result.

Theorem 1.2. Suppose $V$ satisfies $\left(V_{1}\right)$ and $\left(V_{2}\right)$. There hold

(i) $u_{q} \rightarrow u_{0}$ in $\mathcal{H}$ as $q \rightarrow 2_{+}$, where $u_{0} \in H^{1}\left(\mathbb{R}^{2}\right)$ is a global minimizer of $d_{a}(2)$, which is defined in (1.5);

$$
\lim _{q \rightarrow 2_{+}}\left\|v_{q}\right\|_{H^{1}\left(\mathbb{R}^{2}\right)}=\infty
$$

From Theorem 1.2, we see that $v_{q}$ will possibly blow up due to its $H^{1}$ norm tends to infinity. This allows us to study further the asymptotic behavior of $v_{q}$.

Theorem 1.3. Suppose $0<a<a^{*}$ and the potential $V$ satisfies $\left(V_{e}\right)$. Then, for any sequence $\left\{q_{k}\right\}$ with $q_{k} \rightarrow 2_{+}$as $k \rightarrow \infty$, there exist a subsequence of $\left\{q_{k}\right\}$, still denoted by $\left\{q_{k}\right\},\left\{x_{k}\right\} \subset \mathbb{R}^{2}$, and $\beta>0$ such that

$$
\frac{1}{\left\|\nabla v_{q_{k}}\right\|_{2}} v_{q_{k}}\left(\frac{x+x_{k}}{\left\|\nabla v_{q_{k}}\right\|_{2}}\right) \rightarrow \frac{\beta}{\|Q\|_{2}} Q(\beta x)
$$

strongly in $L^{2}\left(\mathbb{R}^{2}\right)$.

The proof of Theorem 1.3 is delicate. In the proof, we will estimate the energy $E_{a, q}\left(v_{q}\right)$ of $v_{q}$. To this end, we need carefully to choose a path and estimate the energy on it. Meanwhile, we find that

$$
E_{a, q}\left(v_{q}\right)=\frac{q-2}{2 q}\left(\frac{2 a_{q}^{*}}{q a}\right)^{\frac{2}{q-2}}+o(1)
$$

as $q \rightarrow 2_{+}$. We remark that $E_{a, q}\left(v_{q}\right) \rightarrow+\infty$ whenever $q \rightarrow 2_{+}$in contrast with the subcritical case, where with the choice of $a>a^{*}$ the energy $E_{a, q}\left(w_{q}\right)$ of the minimizer $w_{q}$ goes to $-\infty$ if $q \rightarrow 2_{-}$, see [12]. Essential difficulties will be encountered in estimating $\int_{\mathbb{R}^{2}}\left|\nabla v_{q}\right|^{2} d x$ and $\int_{\mathbb{R}^{2}} V(x) v_{q}^{2} d x$, which can not be done as simple as the subcritical and critical cases. Moreover, although one expects an estimate for these two terms in the 
supercritical case similar to that for the subcritical and critical cases, it is not able to carry through. Fortunately, we eventually find a suitable estimate enough to serve our purpose.

Finally, we consider a special case $V(x)=|x|^{p}$. In this case, we have a better description of the limiting function.

Corollary 1.2. Suppose $0<a<a^{*}$ and $V(x)=|x|^{p}, p \geq 1$. Then, for any sequence $\left\{q_{k}\right\}$, $q_{k} \rightarrow 2_{+}$as $k \rightarrow \infty$, there exists a subsequence of $\left\{q_{k}\right\}$, still denoted by $\left\{q_{k}\right\}$, such that

$$
\frac{1}{\left\|\nabla v_{q_{k}}\right\|_{2}} v_{q_{k}}\left(\frac{x}{\left\|\nabla v_{q_{k}}\right\|_{2}}\right) \rightarrow \frac{1}{\|Q\|_{2}} Q(x)
$$

strongly in $L^{2}\left(\mathbb{R}^{2}\right)$ and

$$
\lim _{k \rightarrow \infty} \tau_{q_{k}}^{-2}\left\|\nabla v_{q_{k}}\right\|_{2}^{2}=1
$$

This paper is organized as follows. In section 2, we collect and prove some relevant results for future reference. Then, in section 3, we establish the existence of critical points of $E_{a, q}$. Finally, we analyze the asymptotic behavior of these critical points in sections 4 and 5 .

\section{Preliminaries}

In this section, we collect and prove some relevant results for future reference.

For any $q \geq 2$, it is well known that problem (1.8) possesses a unique radially symmetric positive solution $\varphi_{q}$. By Lemma 8.1.2 in [6], $\varphi_{q}$ satisfies

$$
\int_{\mathbb{R}^{2}}\left|\nabla \varphi_{q}\right|^{2} d x=\int_{\mathbb{R}^{2}}\left|\varphi_{q}\right|^{2} d x=\frac{2}{q+2} \int_{\mathbb{R}^{2}}\left|\varphi_{q}\right|^{q+2} d x .
$$

It is known from [5] that there exist positive constants $\delta, C$ and $R_{0}$, independent of $q>0$, such that for any $|x| \geq R_{0}$,

$$
\left|\varphi_{q}(x)\right|+\left|\nabla \varphi_{q}(x)\right| \leq C e^{-\delta|x|} .
$$

Lemma 2.1. Let $\varphi_{q} \geq 0$ be the unique solution of (1.8) with $2 \leq q \leq 3$. Then, $\varphi_{q} \rightarrow Q$ strongly in $H^{1}\left(\mathbb{R}^{2}\right)$ as $q \rightarrow 2_{+}$and there exist positive constants $C$ and $\delta$ independent of $q$ such that

$$
\varphi_{q}(x) \leq C e^{-\delta|x|} \text { for } x \in \mathbb{R}^{2} .
$$

Moreover, $a_{q}^{*} \rightarrow a^{*}=\|Q\|_{2}^{2}$ as $q \rightarrow 2_{+}$, where

$$
a_{q}^{*}=\left\|\varphi_{q}\right\|_{2}^{q} .
$$

Proof. By the Gagliardo-Nirenberg inequality(see [17]), we have

$$
\int_{\mathbb{R}^{2}}|u|^{q+2} d x \leq \frac{q+2}{2\left\|\varphi_{q}\right\|_{2}^{q}}\left(\int_{\mathbb{R}^{2}}|\nabla u|^{2} d x\right)^{\frac{q}{2}} \int_{\mathbb{R}^{2}}|u|^{2} d x
$$


for any $u \in H^{1}\left(\mathbb{R}^{2}\right)$, that is,

$$
\left\|\varphi_{q}\right\|_{2} \leq\left(\frac{q+2}{2}\right)^{\frac{1}{q}} \frac{\left(\int_{\mathbb{R}^{2}}|\nabla u|^{2} d x\right)^{\frac{1}{2}}\left(\int_{\mathbb{R}^{2}}|u|^{2} d x\right)^{\frac{1}{q}}}{\left(\int_{\mathbb{R}^{2}}|u|^{q+2} d x\right)^{\frac{1}{q}}} .
$$

Choosing $\psi \in C_{c}^{\infty}\left(\mathbb{R}^{2}\right)$ such that $0 \leq \psi \leq 1$ and $\psi \neq 0$, we obtain the uniform $L^{2}$ bound of $\varphi_{q}$ in $q$ :

$$
\left\|\varphi_{q}\right\|_{2} \leq\left(\frac{5}{2}\right)^{\frac{1}{2}} \frac{\left(\int_{\mathbb{R}^{2}}|\nabla \psi|^{2} d x+1\right)^{\frac{1}{2}}\left(\int_{\mathbb{R}^{2}}|\psi|^{2} d x+1\right)^{\frac{1}{2}}}{\min \left\{\left(\int_{\mathbb{R}^{2}}|\psi|^{5} d x\right)^{\frac{1}{2}},\left(\int_{\mathbb{R}^{2}}|\psi|^{5} d x\right)^{\frac{1}{3}}\right\}}
$$

Therefore, equation (2.1) implies that $\varphi_{q}$ is uniformly bounded in $H^{1}\left(\mathbb{R}^{2}\right)$ for $2 \leq q \leq 3$. Since $\varphi_{q} \in H_{r a d}^{1}\left(\mathbb{R}^{2}\right)$ and $H_{\text {rad }}^{1}\left(\mathbb{R}^{2}\right) \hookrightarrow L^{p}\left(\mathbb{R}^{2}\right)$ is compact for $p>2$, there exists $\varphi \in$ $H_{\text {rad }}^{1}\left(\mathbb{R}^{2}\right)$ such that $\varphi_{q} \rightarrow \varphi$ weakly in $H_{\text {rad }}^{1}\left(\mathbb{R}^{2}\right)$ and $\varphi_{q} \rightarrow \varphi$ strongly in $L^{p}\left(\mathbb{R}^{2}\right)$ for $p>2$. Thus, we derive for $q \rightarrow 2_{+}$that

$$
\begin{aligned}
& \int_{\mathbb{R}^{2}}\left|\varphi_{q}^{q+2}-\varphi^{4}\right| d x \\
\leq & \int_{\mathbb{R}^{2}}\left|\varphi_{q}^{q+2}-\varphi^{q+2}\right| d x+\int_{\mathbb{R}^{2}}\left|\varphi^{q+2}-\varphi^{4}\right| d x \\
\leq & C\left(\int_{\mathbb{R}^{2}}\left(\left|\varphi_{q}\right|^{q+1}+|\varphi|^{q+1}\right)\left|\varphi_{q}-\varphi\right| d x+\int_{\mathbb{R}^{2}}\left|\varphi^{q+2}-\varphi^{4}\right| d x\right) \\
\leq & C\left[\left(\int_{\mathbb{R}^{2}}\left|\varphi_{q}\right|^{\frac{4(q+1)}{3}}+|\varphi|^{\frac{4(q+1)}{3}} d x\right)^{\frac{3}{4}}\left(\int_{\mathbb{R}^{2}}\left|\varphi_{q}-\varphi\right|^{4} d x\right)^{\frac{1}{4}}+\int_{\mathbb{R}^{2}}\left|\varphi^{q+2}-\varphi^{4}\right| d x\right] \\
\rightarrow & 0 .
\end{aligned}
$$

Observe that $\varphi$ satisfies (1.6). By the Pohozaev identity (2.1), we have

$$
\int_{\mathbb{R}^{2}}|\nabla \varphi|^{2} d x=\int_{\mathbb{R}^{2}}|\varphi|^{2} d x=\frac{1}{2} \int_{\mathbb{R}^{2}}|\varphi|^{4} d x
$$

Hence, we deduce from (2.1), (2.6) and (2.7) that

$$
\int_{\mathbb{R}^{2}}\left|\nabla \varphi_{q}\right|^{2} d x=\frac{2}{q+2} \int_{\mathbb{R}^{2}}\left|\varphi_{q}\right|^{q+2} d x \rightarrow \frac{1}{2} \int_{\mathbb{R}^{2}}|\varphi|^{4} d x=\int_{\mathbb{R}^{2}}|\nabla \varphi|^{2} d x
$$

which implies that $\varphi_{q} \rightarrow \varphi$ in $H^{1}\left(\mathbb{R}^{2}\right)$. By the uniqueness of positive solutions to (1.6), we have $\varphi=Q$. Applying the standard elliptic theory, we may show that $\varphi_{q}$ is uniformly bounded in $L^{\infty}\left(\mathbb{R}^{2}\right)$. So by $(2.2)$, there exists $C, \delta>0$ independent of $q$ such that

$$
\varphi_{q}(x) \leq C e^{-\delta|x|}
$$

for $x \in \mathbb{R}^{2}$.

In order to find the critical points of the constrained problem, it needs, among other things, to find a $(P S)$ sequence on the constrained manifold. To bound the $(P S)$ sequence, we need the following variant mountain pass theorem. 
Let $\mathcal{E}_{a, q}: \mathcal{H} \times \mathbb{R} \rightarrow \mathbb{R}$ be the functional

$$
\mathcal{E}_{a, q}(u, s)=E_{a, q}(H(u, s))=\frac{1}{2} e^{2 s} \int_{\mathbb{R}^{2}}\left[|\nabla u|^{2}+V\left(e^{-s} x\right)|u|^{2}\right] d x-\frac{a}{q+2} e^{s q} \int_{\mathbb{R}^{2}}|u|^{q+2} d x,
$$

where $H(u, s)=e^{s} u\left(e^{s} x\right)$. For $\varphi_{1}, \varphi_{2} \in S(1)$ being nonnegative, we define

$$
\mathcal{P}_{q}=\left\{\gamma \in C([0,1], S(1) \times \mathbb{R}): \gamma(0)=\left(\varphi_{1}, 0\right), \gamma(1)=\left(\varphi_{2}, 0\right)\right\}
$$

and

$$
b_{q}=\inf _{\gamma \in \mathcal{P}_{q}} \max _{t \in[0,1]} \mathcal{E}_{a, q}(\gamma(t)) .
$$

Let $c_{q}$ be the mountain pass level for $E_{a, q}$ defined by

$$
c_{q}=\inf _{g \in \Gamma_{q}} \max _{t \in[0,1]} E_{a, q}(g(t)),
$$

where

$$
\Gamma_{q}=\left\{g \in C([0,1], S(1)) \mid g(0)=\varphi_{1}, g(1)=\varphi_{2}\right\}
$$

Since $H\left(\mathcal{P}_{q}\right) \subset \Gamma_{q}$ and $(g, 0) \in \mathcal{P}_{q}$ for any $g \in \Gamma_{q}$, we have the following result.

Lemma 2.2. There holds $b_{q}=c_{q}$.

Let us denote by $Y$ the space $\mathcal{H} \times \mathbb{R}$ with the norm $\|\cdot\|_{Y}^{2}=\|\cdot\|_{\mathcal{H}}^{2}+\|\cdot\|_{\mathbb{R}}^{2}$ and denote by $Y^{-1}$ its dual space.

Proposition 2.1. Suppose that

$$
b_{q}=\inf _{\gamma \in \mathcal{P}_{q}} \max _{t \in[0,1]} \mathcal{E}_{a, q}(\gamma(t))>\sup \left\{\mathcal{E}_{a, q}(\gamma(0)), \mathcal{E}_{a, q}(\gamma(1))\right\} .
$$

Then, there exist $\left\{\gamma_{n}\right\}:=\left\{\left(g_{n}, 0\right)\right\} \subset \mathcal{P}_{q}$ with $g_{n} \geq 0$ and $\left\{\left(w_{n}, s_{n}\right)\right\} \subset S(1) \times \mathbb{R}$ such that

$$
\begin{gathered}
\lim _{n \rightarrow \infty} \sup _{\gamma_{n}} \mathcal{E}_{a, q}=b_{q} ; \\
\lim _{n \rightarrow \infty} \mathcal{E}_{a, q}\left(w_{n}, s_{n}\right)=b_{q} ; \\
\left\|\left.\mathcal{E}_{a, q}^{\prime}\right|_{S(1) \times \mathbb{R}}\left(w_{n}, s_{n}\right)\right\|_{Y^{-1}} \rightarrow 0 ; \\
\lim _{n \rightarrow \infty} \operatorname{dist}\left(\left(w_{n}, s_{n}\right),\left(g_{n}, 0\right)\right)=0 .
\end{gathered}
$$

Proof. By Lemma 2.3, there exists $h_{n} \in \Gamma_{q}$ such that $\lim _{n \rightarrow \infty} \sup _{h_{n}} E_{a, q}\left(h_{n}\right)=c_{q}=b_{q}$. Obviously, $\left|h_{n}\right| \in \Gamma_{q}$ and $c_{q} \leq E_{a, q}\left(\left|h_{n}\right|\right) \leq E_{a, q}\left(h_{n}\right)$. Hence, $\lim _{n \rightarrow \infty} \sup _{h_{n}} E_{a, q}\left(\left|h_{n}\right|\right)=b_{q}$ and $\lim _{n \rightarrow \infty} \sup _{\left(\left|h_{n}\right|, 0\right)} \mathcal{E}_{a, q}=b_{q}$. The conclusion follows from Theorem 3.2 in [8], where we choose $\varphi=\mathcal{E}_{a, q}, \mathcal{F}=\mathcal{P}_{q}, B=(\gamma(0), \gamma(1)), c=b_{q}, X=S(1) \times \mathbb{R}, \gamma_{n}=\left(g_{n}, 0\right)$ with $g_{n}=\left|h_{n}\right|$.

Finally, we have the following Pohozaev identity. 
Lemma 2.3. If $u$ solves

$$
-\Delta u+V(x) u=a u^{q+1}+\mu u
$$

then

$$
\int_{\mathbb{R}^{2}}|\nabla u|^{2} d x-\frac{1}{2} \int_{\mathbb{R}^{2}} x \cdot \nabla V|u|^{2} d x=\frac{q a}{q+2} \int_{\mathbb{R}^{2}}|u|^{q+2} d x .
$$

Proof. It is known from [1] that

$$
\int_{\mathbb{R}^{2}} V|u|^{2} d x+\frac{1}{2} \int_{\mathbb{R}^{2}} x \cdot \nabla V|u|^{2} d x=\frac{2 a}{q+2} \int_{\mathbb{R}^{2}}|u|^{q+2} d x+\mu \int_{\mathbb{R}^{2}}|u|^{2} d x .
$$

Multiplying (2.18) by $u$ and integrating by part, we have

$$
\int_{\mathbb{R}^{2}}|\nabla u|^{2} d x+\int_{\mathbb{R}^{2}} V|u|^{2} d x=a \int_{\mathbb{R}^{2}}|u|^{q+2} d x+\mu \int_{\mathbb{R}^{2}}|u|^{2} d x .
$$

The Pohozaev identity (2.19) follows from (2.20) and (2.21).

\section{Existence}

In this section, we show the existence of two critical points of the functional $E_{a, q}(u)$ on the sphere $S(1)$ defined in (1.11). The first critical point of $E_{a, q}(u)$ will be found as a minimizer of the minimization problem

$$
m_{k}=\inf _{u \in A_{k}} E_{a, q}(u)
$$

where

$$
A_{k}=\left\{\left.u \in S(1)\left|\int_{\mathbb{R}^{2}}\right| \nabla u\right|^{2} d x<k\right\} .
$$

Once $m_{k}$ is achieved, it is necessary to show that the minimizer is not on the boundary of $A_{k}$ :

$$
\partial A_{k}=\left\{\left.u \in S(1)\left|\int_{\mathbb{R}^{2}}\right| \nabla u\right|^{2} d x=k\right\} .
$$

The minimizer is then a critical point of $E_{a, q}(u)$. The second critical point of $E_{a, q}(u)$ is obtained by the mountain pass theorem.

At the beginning, we recall the following compactness lemma, which can be proved as that in [18].

Lemma 3.1. Suppose $V \in L_{l o c}^{\infty}\left(\mathbb{R}^{2}\right)$ and $\lim _{x \rightarrow \infty} V(x)=\infty$. Then the embedding $\mathcal{H} \hookrightarrow$ $L^{p}\left(\mathbb{R}^{2}\right)$ is compact for any $p \in[2, \infty)$.

Now, we show that there is a minimizer of $m_{k}$, which is a critical point of $E_{a, q}(u)$. In the sequel, we denote $f^{t}(x)=t f(t x)$ for any function $f$. 
Proposition 3.1. Suppose $\left(V_{1}\right)$ and $\left(V_{2}\right)$. For each $a \in\left(0, a^{*}\right)$, there exists a positive critical point $u_{q} \in A_{\tau_{q}^{2}}$ of the functional $E_{a, q}(u)$ such that

$$
E_{a, q}\left(u_{q}\right)=\inf _{u \in A_{\tau_{q}^{2}}} E_{a, q}(u)
$$

if $q>2$ close to 2 , where $\tau_{q}^{2}$ is defined in (1.12).

Proof. We consider the minimization problem

$$
m_{k}=\inf _{u \in A_{k}} E_{a, q}(u) .
$$

Fix $k>0$, we claim that $m_{k}$ is achieved. Indeed, let $\left\{u_{n}\right\} \subset A_{k}$ be a minimizing sequence of $m_{k}$, which is obviously bounded in $\mathcal{H}$. We can assume that it converges weakly to $u_{k} \in \mathcal{H}$. By Lemma 3.1, we have $u_{k} \in S(1)$. The lower semi-continuity of the functional $E_{a, q}$ implies

$$
E_{a, q}\left(u_{k}\right) \leq \liminf _{n \rightarrow \infty} E_{a, q}\left(u_{n}\right)=m_{k} .
$$

Therefore, $E_{a, q}\left(u_{k}\right)=m_{k}$ and $\left\|\nabla u_{k}\right\|_{2}^{2} \leq k$. That is, $u_{k}$ is a minimizer of $m_{k}$.

Next, we show that $u_{k}$ is a critical point of $E_{a, q}(u)$. It is sufficient to prove $u_{k} \notin \partial A_{k}$. Now, we will find a suitable $k>0$ so that $u_{k}$ belongs to $A_{k}$. This will be done if we can find an element $\varphi \in A_{k}$ so that

$$
E_{a, q}(\varphi)<\inf _{u \in \partial A_{k}} E_{a, q}(u) .
$$

In the following, we show that inequality (3.2) is valid for $k=\tau_{q}^{2}$, where $\tau_{q}$ is given in (1.12). By the Gagliardo-Nirenberg inequality[17], we have

$$
\int_{\mathbb{R}^{2}}|u|^{q+2} d x \leq \frac{q+2}{2 a_{q}^{*}}\left(\int_{\mathbb{R}^{2}}|\nabla u|^{2} d x\right)^{\frac{q}{2}} \int_{\mathbb{R}^{2}} u^{2} d x
$$

where $a_{q}^{*}$ is defined in (2.4). This implies that for any $u \in S(1)$,

$$
\left.E_{a, q}\right|_{V=0}(u) \geq \frac{1}{2} \int_{\mathbb{R}^{2}}|\nabla u|^{2} d x-\frac{a}{2 a_{q}^{*}}\left(\int_{\mathbb{R}^{2}}|\nabla u|^{2} d x\right)^{\frac{q}{2}},
$$

where $\left.E_{a, q}\right|_{V=0}(u)$ denotes the functional obtained by taking $V \equiv 0$ in $E_{a, q}$. In view of (3.4), we consider the function $g: \mathbb{R} \mapsto \mathbb{R}$ defined by

$$
g(s)=\frac{1}{2} s-\frac{a}{2 a_{q}^{*}} s^{\frac{q}{2}} .
$$

We may verify that $g$ is increasing in $\left(0, \tau_{q}^{2}\right)$ and decreasing in $\left(\tau_{q}^{2}, \infty\right)$. Therefore, the function $g$ attains its maximum at $s=\tau_{q}^{2}$, and

$$
g\left(\tau_{q}^{2}\right)=\frac{q-2}{2 q} \tau_{q}^{2}
$$

Since $a<a^{*}$ and by Lemma $2.1 a_{q}^{*} \rightarrow a^{*}$ as $q \rightarrow 2$, we remark that

$$
\frac{q-2}{2 q} \tau_{q}^{2} \rightarrow+\infty \text { as } q \rightarrow 2_{+}
$$


By (3.4), we have

$$
\inf _{u \in \partial A_{\tau_{q}^{2}}} E_{a, q}(u) \geq\left.\inf _{u \in \partial A_{\tau_{q}^{2}}} E_{a, q}\right|_{V=0}(u) \geq \frac{q-2}{2 q} \tau_{q}^{2}=: \theta \tau_{q}^{2} .
$$

Apparently, if $u \in A_{\frac{1}{4} \theta \tau_{q}^{2}}$, then

$$
\left.E_{a, q}\right|_{V=0}(u) \leq \frac{1}{8} \theta \tau_{q}^{2}
$$

Choose $\varphi$ such that $\varphi \in C_{c}^{\infty}\left(\mathbb{R}^{2}\right), \varphi \geq 0$, and $\|\varphi\|_{2}^{2}=1$ and let

$$
t_{0}=t_{0}(q)=\frac{1}{4} \theta \tau_{q}^{2}\left(\int_{\mathbb{R}^{2}}|\nabla \varphi|^{2} d x\right)^{-\frac{1}{2}}
$$

be such that

$$
\int_{\mathbb{R}^{2}}\left|\nabla \varphi^{t_{0}}\right|^{2} d x=\frac{1}{4} \theta \tau_{q}^{2}
$$

Since $\tau_{q} \rightarrow \infty$ as $q \rightarrow 2_{+}$, so does $t_{0}$. By Lemma 2.1 and the Lebesgue dominated convergence theorem,

$$
\int_{\mathbb{R}^{2}} V(x)\left|\varphi^{t_{0}}(x)\right|^{2} d x=\int_{\mathbb{R}^{2}} V\left(\frac{x}{t_{0}}\right)|\varphi(x)|^{2} d x \rightarrow V(0)
$$

as $q \rightarrow 2_{+}$. It follows from (3.9) and (3.10) that

$$
E_{a, q}\left(\varphi^{t_{0}}\right) \leq \frac{1}{8} \theta \tau_{q}^{2}+2 V(0)
$$

We deduce from (3.6) that

$$
\inf _{u \in \partial A_{\tau_{q}^{2}}} E_{a, q}(u)-E_{a, q}\left(\varphi^{t_{0}}\right) \geq \frac{7}{8} \theta \tau_{q}^{2}-2 V(0)>0
$$

for $q>2$ and close to 2 . Hence, we have $\varphi^{t_{0}} \in A_{\tau_{q}^{2}}$ and

$$
m_{\tau_{q}^{2}} \leq E_{a, q}\left(\varphi^{t_{0}}\right)<\inf _{u \in \partial A_{\tau_{q}^{2}}} E_{a, q}(u)
$$

for $q>2$ and close to 2. Consequently, $E_{a, q}(u)$ attains its minimum at $u_{\tau_{q}^{2}} \in A_{\tau_{q}^{2}}$ for $q$ close to 2. Note that $E_{a, q}(|u|) \leq E_{a, q}(u)$, we can assume that $u_{k}$ is nonnegative. In addition, $u_{\tau_{q}^{2}}$ solves (1.10) for some Lagrange multiplier $\mu_{q}$. By the strong maximum principle, $u_{\tau_{q}^{2}}>0$. The proof is complete.

Once we show that the functional $E_{a, q}(u)$ has a mountain-pass geometry, we may find a $(P S)$ sequence of $E_{a, q}(u)$, which is close to the Pohozaev manifold. Indeed, we have the following lemma, which is motivated by $[5,8,13]$.

Lemma 3.2. Suppose

$$
c_{q}>\max \left\{E_{a, q}\left(\varphi_{1}\right), E_{a, q}\left(\varphi_{2}\right)\right\}
$$


where $c_{q}$ is defined in (2.11). Then, there is a sequence $\left\{u_{n}\right\} \subset S(1)$ such that

$$
\begin{array}{r}
E_{a, q}\left(u_{n}\right) \rightarrow c_{q}, \\
\left\|\left.E_{a, q}^{\prime}\right|_{S(1)}\left(u_{n}\right)\right\|_{\mathcal{H}^{-1}} \rightarrow 0, \\
Q_{q}\left(u_{n}\right) \rightarrow 0, \text { as } n \rightarrow \infty,
\end{array}
$$

where

$$
Q_{q}(u)=\int_{\mathbb{R}^{2}}|\nabla u|^{2} d x-\frac{1}{2} \int_{\mathbb{R}^{2}} x \cdot \nabla V|u|^{2} d x-\frac{q a}{q+2} \int_{\mathbb{R}^{2}}|u|^{q+2} d x
$$

and $\mathcal{H}^{-1}$ denotes the dual space of $\mathcal{H}$.

Moreover, there is a sequence $v_{n} \in S(1)$ with $v_{n} \geq 0$ such that

$$
\lim _{n \rightarrow \infty}\left\|u_{n}-v_{n}\right\|_{\mathcal{H}}=0 .
$$

Proof. By Lemma 2.2, we have $b_{q}=c_{q}$. Hence, equation (3.12) implies that equation (2.13) holds true, so do the results in Proposition 3.1.

Let $u_{n}=H\left(w_{n}, s_{n}\right)$. By $(2.15)$ and Lemma 2.2, we have

$$
E_{a, q}\left(u_{n}\right)=\mathcal{E}_{a, q}\left(w_{n}, s_{n}\right) \rightarrow c_{q} .
$$

By (2.16),

$$
\left\langle\mathcal{E}_{a, q}^{\prime}\left(w_{n}, s_{n}\right), z\right\rangle_{Y^{-1}, Y}=o\left(\|z\|_{Y}\right),
$$

for all $z \in \mathcal{T}_{\left(w_{n}, s_{n}\right)}:=\left\{\left(z_{1}, z_{2}\right) \in Y:\left\langle w_{n}, z_{1}\right\rangle_{2}=0\right\}$.

Choosing $z=(0,1)$ in $(3.16)$, we find

$$
\begin{aligned}
& \left\langle\mathcal{E}_{a, q}^{\prime}\left(w_{n}, s_{n}\right),(0,1)\right\rangle \\
& =\left.\frac{d}{d t} \mathcal{E}_{a, q}\left(w_{n}, s_{n}+t\right)\right|_{t=0} \\
& =\left.\frac{d}{d t} E_{a, q}\left(H\left(w_{n}, s_{n}+t\right)\right)\right|_{t=0} \\
& =\left.\frac{d}{d t} E_{a, q}\left(e^{s_{n}+t} w_{n}\left(e^{s_{n}+t} x\right)\right)\right|_{t=0} \\
& =e^{2 s_{n}} \int_{\mathbb{R}^{2}}\left|\nabla w_{n}\right|^{2} d x-\frac{1}{2} \int_{\mathbb{R}^{2}} \nabla V\left(e^{-s_{n}} x\right) \cdot e^{-s_{n}} x\left|w_{n}\right|^{2} d x-\frac{q a}{q+2} e^{q s_{n}} \int_{\mathbb{R}^{2}}\left|w_{n}\right|^{q+2} d x \\
& =\int_{\mathbb{R}^{2}}\left|\nabla u_{n}\right|^{2} d x-\frac{1}{2} \int_{\mathbb{R}^{2}} x \cdot \nabla V\left|u_{n}\right|^{2} d x-\frac{q a}{q+2} \int_{\mathbb{R}^{2}}\left|u_{n}\right|^{q+2} d x \\
& =Q_{q}\left(u_{n}\right) \rightarrow 0,
\end{aligned}
$$

as $n \rightarrow \infty$. 
For any $\varphi \in T_{u_{n}}:=\left\{u_{n} \in \mathcal{H},\left\langle u_{n}, \varphi\right\rangle_{2}=0\right\}$, setting $\psi=e^{-s_{n}} \varphi\left(e^{-s_{n}} x\right)$,

$$
\begin{aligned}
\left\langle\left. E_{a, q}^{\prime}\right|_{S(1)}\left(u_{n}\right), \varphi\right\rangle & =\left\langle E_{a, q}^{\prime}\left(u_{n}\right), \varphi\right\rangle \\
& =\left.\frac{d}{d t} E_{a, q}\left(u_{n}+t \varphi\right)\right|_{t=0} \\
& \left.=\frac{d}{d t} E_{a, q}\left(e^{s_{n}} w_{n}\left(e^{s_{n}} x\right)\right)+t e^{s_{n}} \psi\left(e^{s_{n}} x\right)\right)\left.\right|_{t=0} \\
& =\left.\frac{d}{d t} E_{a, q}\left(H\left(w_{n}+t \psi, s_{n}\right)\right)\right|_{t=0} \\
& =\left.\frac{d}{d t} \mathcal{E}_{a, q}\left(w_{n}+t \psi, s_{n}\right)\right|_{t=0} \\
& =\left\langle\mathcal{E}_{a, q}^{\prime}\left(w_{n}, s_{n}\right),(\psi, 0)\right\rangle .
\end{aligned}
$$

Since $\left\langle w_{n}, \psi\right\rangle_{2}=\left\langle u_{n}, \varphi\right\rangle_{2}=0,(\psi, 0) \in \mathcal{T}_{\left(w_{n}, s_{n}\right)}$. By (2.16) and (3.17), we have

$$
\left\langle\left. E_{a, q}^{\prime}\right|_{S(1)}\left(u_{n}\right), \varphi\right\rangle=o\left(\|\psi\|_{\mathcal{H}}\right),
$$

and (2.17) implies

$$
\left|s_{n}\right| \leq \operatorname{dist}\left(\left(w_{n}, s_{n}\right),\left(g_{n}, 0\right)\right) \rightarrow 0
$$

as $n \rightarrow \infty$. Hence, $\|\psi\|_{\mathcal{H}} \leq 4\|\varphi\|_{\mathcal{H}}$. It results

$$
\left\langle\left. E_{a, q}^{\prime}\right|_{S(1)}\left(u_{n}\right), \varphi\right\rangle=o\left(\|\varphi\|_{\mathcal{H}}\right) .
$$

By $(2.17)$ and (3.19), we have $\lim _{n \rightarrow \infty} \operatorname{dist}_{\mathcal{H}}\left(w_{n}, g_{n}\right)=0$. So we may choose $t_{n} \in[0,1]$ such that

$$
\left\|w_{n}-g_{n}\left(t_{n}\right)\right\|_{\mathcal{H}}=\operatorname{dist}_{\mathcal{H}}\left(w_{n}, g_{n}\right)
$$

Let $v_{n}=g_{n}\left(t_{n}\right) \geq 0$. We have $\lim _{n \rightarrow \infty}\left\|w_{n}-v_{n}\right\|_{\mathcal{H}}=0$. This with (3.19) yields

$$
\lim _{n \rightarrow \infty}\left\|u_{n}-v_{n}\right\|_{\mathcal{H}}=\lim _{n \rightarrow \infty}\left\|H\left(w_{n}, s_{n}\right)-v_{n}\right\|_{\mathcal{H}}=\lim _{n \rightarrow \infty}\left\|w_{n}-v_{n}\right\|_{\mathcal{H}}=0 .
$$

Now, we seek for the second critical point of $E_{a, q}(u)$ by the variant mountain pass theorem.

Proposition 3.2. Suppose $\left(V_{1}\right)$ and $\left(V_{2}\right)$ hold. If $q>2$ and close to 2 , then $E_{a, q}(u)$ admits a second critical point $v_{q}$ on $S(1)$ at the mountain pass level.

Proof. First, we verify that $E_{a, q}(u)$ has a mountain pass geometry on $S(1)$.

Let $\varphi \in C_{c}^{\infty}\left(\mathbb{R}^{2}\right)$ be such that $\varphi \geq 0$, and $\|\varphi\|_{2}^{2}=1$. Denote $\varphi^{t}(x)=t \varphi(t x)$. We find

$$
E_{a, q}\left(\varphi^{t}\right)=\frac{1}{2} t^{2} \int_{\mathbb{R}^{2}}|\nabla \varphi|^{2} d x+\frac{1}{2} \int_{\mathbb{R}^{2}} V\left(\frac{x}{t}\right) \varphi^{2} d x-\frac{a t^{q}}{q+2} \int_{\mathbb{R}^{2}}|\varphi|^{q+2} d x .
$$

Since

$$
\int_{\mathbb{R}^{2}} V\left(\frac{x}{t}\right) \varphi^{2} d x \rightarrow V(0) \text { as } t \rightarrow \infty
$$

and $q>2$, we have

$$
E_{a, q}\left(\varphi^{t}\right) \rightarrow-\infty \text { as } t \rightarrow \infty
$$


Taking

$$
t_{1}=C 2^{\frac{1}{(q-2)^{2}}}
$$

with $C$ large enough and independent of $q$ such that $E_{a, q}\left(\varphi^{t_{1}}\right)<0$ for $q>2$ and close to 2, we define

$$
\Gamma_{q}=\left\{g \in C([0,1], S(1)) \mid g(0)=\varphi^{t_{0}}, g(1)=\varphi^{t_{1}}\right\} .
$$

By the choice of $t_{1}$ and (3.11), we see that (3.12) is valid. This means that $E_{a, q}$ has the mountain pass geometry. By Lemma 3.2, there is a sequence $\left\{u_{n}\right\} \subset S(1)$ such that

$$
\begin{array}{r}
E_{a, q}\left(u_{n}\right) \rightarrow c_{q}, \\
\left\|\left.E_{a, q}^{\prime}\right|_{S(1)}\left(u_{n}\right)\right\|_{\mathcal{H}^{-1}} \rightarrow 0, \\
Q_{q}\left(u_{n}\right) \rightarrow 0, \text { as } n \rightarrow \infty .
\end{array}
$$

Hence, (3.13), the identity

$$
\frac{q-2}{2} \int_{\mathbb{R}^{2}}|\nabla u|^{2} d x+\frac{1}{2} \int_{\mathbb{R}^{2}}(q V+x \cdot \nabla V) u^{2} d x=q E_{a, q}(u)-Q_{q}(u)
$$

and $\left(V_{2}\right)$ give that the sequence $\left\{u_{n}\right\}$ is bounded in $\mathcal{H}$. So there exists $v_{q} \in \mathcal{H}$ such that $u_{n} \rightarrow v_{q}$ weakly in $\mathcal{H}$. Since $\left.E_{a, q}^{\prime}\right|_{S(1)}\left(u_{n}\right) \rightarrow 0$ in $\mathcal{H}^{-1}$, by Lemma 3 in [5], there is a $\mu_{q}^{n}$ such that

$$
-\Delta u_{n}+V u_{n}-a\left|u_{n}\right|^{q} u_{n}-\mu_{q}^{n} u_{n} \rightarrow 0 \quad \text { in } \quad \mathcal{H}^{-1}
$$

Hence,

$$
\mu_{q}^{n}=\int_{\mathbb{R}^{2}}\left|\nabla u_{n}\right|^{2} d x+\int_{\mathbb{R}^{2}} V\left|u_{n}\right|^{2} d x-a \int_{\mathbb{R}^{2}}\left|u_{n}\right|^{q+2} d x+o\left(\left\|u_{n}\right\|_{\mathcal{H}}\right),
$$

which is bounded. Without of the loss of generality, we may assume $\mu_{q}^{n} \rightarrow \mu_{q}$ as $n \rightarrow \infty$. It yields

$$
-\Delta v_{q}+V v_{q}-a\left|v_{q}\right|^{q} v_{q}-\mu_{q} v_{q}=0 .
$$

We deduce from (3.25) and (3.26) that

$$
\int_{\mathbb{R}^{2}}\left|\nabla u_{n}\right|^{2} d x+\int_{\mathbb{R}^{2}} V u_{n}^{2} d x-a \int_{\mathbb{R}^{2}}\left|u_{n}\right|^{q+2} d x-\mu_{q}^{n}=o\left(\left\|u_{n}\right\|_{\mathcal{H}}\right)
$$

and

$$
\int_{\mathbb{R}^{2}}\left|\nabla v_{q}\right|^{2} d x+\int_{\mathbb{R}^{2}} V\left|v_{q}\right|^{2} d x-a \int_{\mathbb{R}^{2}}\left|v_{q}\right|^{q+2} d x-\mu_{q}=0 .
$$

By the Brézis-Lieb lemma,

$$
\int_{\mathbb{R}^{2}}\left|\nabla u_{n}-\nabla v_{q}\right|^{2} d x+\int_{\mathbb{R}^{2}} V\left|u_{n}-v_{q}\right|^{2} d x=a \int_{\mathbb{R}^{2}}\left|u_{n}-v_{q}\right|^{q+2} d x+o\left(\left\|u_{n}\right\|_{\mathcal{H}}\right) .
$$

Since $\mathcal{H} \hookrightarrow L^{q}\left(\mathbb{R}^{2}\right)$ is compact for any $q \geq 2$, we have

$$
u_{n} \rightarrow v_{q} \text { in } \mathcal{H} \text {. }
$$

Thus, $E_{a, q}\left(v_{q}\right)=c_{q}$ and $\left\|v_{q}\right\|_{2}=1$. Furthermore, by Lemma 3.2, $\lim _{n \rightarrow \infty}\left\|v_{n}-v_{q}\right\|_{\mathcal{H}}=0$. Noting $v_{n} \geq 0$ and $c_{q}>0$, we have $v_{q} \geq 0$ and $v_{q} \neq 0$. By the strong maximum principle, we conclude $v_{q}>0$. This ends the proof. 
Proof of Theorem 1.1. The results in Theorem 1.1 follow by Propositions 3.1 and 3.2.

Now, we study the asymptotic behavior of critical points $u_{q}$ and $v_{q}$ as $q \rightarrow 2_{+}$.

Lemma 3.3. The minimizers $\left\{u_{q}\right\}$ is uniformly bounded in $\mathcal{H}$ for $q>2$ close to 2.

Proof. We argue indirectly. Suppose $\left\{u_{q}\right\}$ is not uniformly bounded, there would exist $\left\{q_{k}\right\}$ with $q_{k} \rightarrow 2_{+}$as $k \rightarrow \infty$ such that

$$
\lim _{k \rightarrow \infty}\left\|u_{q_{k}}\right\|_{\mathcal{H}}^{2}=+\infty
$$

Let $\varphi \in C_{c}^{\infty}\left(\mathbb{R}^{2}\right)$ be such that

$$
0 \leq \varphi \leq 1 \text { and }\|\varphi\|_{2}^{2}=1 .
$$

Since $\tau_{q}^{2}$ given in (1.12) tends to infinity as $q \rightarrow 2_{+}$, we have $\varphi \in A_{\tau_{q}^{2}}$, for $q>2$ close to 2 , and

$$
E_{a, q}\left(u_{q}\right)=\inf _{u \in A_{\tau_{q}^{2}}} E_{a, q}\left(u_{q}\right) \leq E_{a, q}(\varphi) \leq \frac{1}{2} \int_{\mathbb{R}^{2}}\left(|\nabla \varphi|^{2}+V|\varphi|^{2}\right) d x .
$$

By Lemma 2.3, $Q_{q}\left(u_{q}\right)=0$, we find from (3.24) and (3.29) that

$$
\begin{aligned}
& \frac{q_{k}-2}{2} \int_{\mathbb{R}^{2}}\left|\nabla u_{q_{k}}\right|^{2} d x+\frac{1}{2} \int_{\mathbb{R}^{2}} V u_{q_{k}}^{2} d x+\frac{1}{2} \int_{\mathbb{R}^{2}}[(q-1) V+x \cdot \nabla V] u_{q_{k}}^{2} d x \\
& =q_{k} E_{a, q_{k}} \leq \frac{3}{2} \int_{\mathbb{R}^{2}}\left(|\nabla \varphi|^{2}+V \varphi^{2}\right) d x .
\end{aligned}
$$

The assumption (V2) implies

$$
\int_{\mathbb{R}^{2}} V u_{q_{k}}^{2} d x \leq C
$$

and

$$
\int_{\mathbb{R}^{2}}\left|\nabla u_{q_{k}}\right|^{2} d x \leq \frac{C}{q_{k}-2} .
$$

By (3.28) and (3.30), we have

$$
\lim _{k \rightarrow \infty}\left\|\nabla u_{q_{k}}\right\|_{2}^{2}=+\infty
$$

Let

$$
\eta_{q_{k}}=\left\|\nabla u_{q_{k}}\right\|_{2}^{-1}
$$

and

Then

$$
\tilde{f}_{q_{k}}(x)=\eta_{q_{k}} u_{q_{k}}\left(\eta_{q_{k}} x\right)
$$

$$
\left\|\nabla \tilde{f}_{q_{k}}\right\|_{2}^{2}=\left\|\tilde{f}_{q_{k}}\right\|_{2}^{2}=1
$$

By $(V 2)$ and (3.30), we have

$$
\left|\int_{\mathbb{R}^{2}} x \cdot \nabla V u_{q_{k}}^{2} d x\right| \leq \int_{\mathbb{R}^{2}}|x \cdot \nabla V| u_{q_{k}}^{2} d x \leq \int_{\mathbb{R}^{2}} C(V+1) u_{q_{k}}^{2} d x \leq C,
$$

and Lemma 2.3 implies

$$
\int_{\mathbb{R}^{2}}\left|\nabla u_{q_{k}}\right|^{2} d x-\frac{1}{2} \int_{\mathbb{R}^{2}} x \cdot \nabla V\left|u_{q_{k}}\right|^{2} d x=\frac{a q_{k}}{q_{k}+2} \int_{\mathbb{R}^{2}}\left|u_{q_{k}}\right|^{q_{k}+2} d x .
$$


This with (3.32) and (3.36) yields that

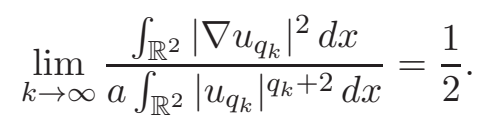

By (3.32), for $k$ large enough, $\left\|\nabla u_{q_{k}}\right\|_{2}^{2} \geq 1$, we deduce from (3.31) and (3.33) that

$$
1 \geq \eta_{q_{k}}^{q_{k}-2}=\left\|\nabla u_{q_{k}}\right\|_{2}^{2-q_{k}} \geq\left(\frac{C}{q_{k}-2}\right)^{\frac{2-q_{k}}{2}} \rightarrow 1 \text { as } k \rightarrow \infty .
$$

Hence

$$
\eta_{q_{k}}^{q_{k}-2} \rightarrow 1 \text { as } k \rightarrow \infty .
$$

It follows from (3.33), (3.38) and (3.39) that there exist $C_{1}>0$ and $C_{2}>0$ such that

$$
C_{1} \leq \int_{\mathbb{R}^{2}}\left|\tilde{f}_{q_{k}}\right|^{q_{k}+2} d x=\eta_{q_{k}}^{q_{k}-2} \frac{\int_{\mathbb{R}^{2}}\left|u_{q_{k}}\right|^{q_{k}+2} d x}{\int_{\mathbb{R}^{2}}\left|\nabla u_{q_{k}}\right|^{2} d x} \leq C_{2}
$$

We claim from (3.35) and (3.40) that, there exist $\left\{y_{q_{k}}\right\} \subset \mathbb{R}^{2}, R_{0}>0$ and $\eta>0$ such that

$$
\liminf _{k \rightarrow \infty} \int_{B_{R_{0}}\left(y_{q_{k}}\right)}\left|\tilde{f}_{q_{k}}\right|^{2} d x \geq \eta
$$

Indeed, if it is not the case, for any $R>0$, there would exist a sequence, still denoted by $\left\{\tilde{f}_{q_{k}}\right\}$ such that

$$
\lim _{k \rightarrow \infty} \sup _{y \in \mathbb{R}^{2}} \int_{B_{R}(y)}\left|\tilde{f}_{q_{k}}\right|^{2} d x=0 .
$$

By the vanishing lemma, see for instance Lemma 1.21 in [20], we have $\tilde{f}_{q_{k}} \rightarrow 0$ strongly in $L^{\gamma}\left(\mathbb{R}^{2}\right)$ for any $\gamma>2$, which contradicts (3.40).

Denote $f_{q_{k}}=\tilde{f}_{q_{k}}\left(x+y_{q_{k}}\right)=\eta_{q_{k}} u_{q_{k}}\left(\eta_{q_{k}}\left(x+y_{q_{k}}\right)\right)$. We have

$$
\begin{aligned}
& \left\|\nabla f_{q_{k}}\right\|_{2}^{2}=\left\|f_{q_{k}}\right\|_{2}^{2}=1 ; \\
& C_{1} \leq \int_{\mathbb{R}^{2}}\left|f_{q_{k}}\right|^{q_{k}+2} d x \leq C_{2} ; \\
& \liminf _{k \rightarrow \infty} \int_{B_{R_{0}}(0)}\left|f_{q_{k}}\right|^{2} d x \geq \eta .
\end{aligned}
$$

Hence, there exist a sequence $\left\{q_{k}\right\}$ and $f \in H^{1}\left(\mathbb{R}^{2}\right)$ such that $f_{q_{k}} \rightarrow f$ weakly in $H^{1}\left(\mathbb{R}^{2}\right)$ and $f_{q_{k}} \rightarrow f$ strongly in $L_{l o c}^{\gamma}\left(\mathbb{R}^{2}\right)$ for any $\gamma \geq 2$. Noting that by $(3.42), f \neq 0$. Since $u_{q_{k}}$ solves (1.11) for the Lagrange multiplier $\mu_{q_{k}}$, we see that $f_{q_{k}}$ solves

$$
-\Delta f_{q_{k}}+\eta_{q_{k}}^{2} V\left(\eta_{q_{k}}\left(x+y_{q_{k}}\right)\right) f_{q_{k}}=\eta_{q_{k}}^{2} \mu_{q_{k}} f_{q_{k}}+\eta_{q_{k}}^{2-q} a f_{q_{k}}^{q_{k}+1} .
$$

Next, we prove that $\left\{\eta_{q_{k}} y_{q_{k}}\right\}$ is uniformly bounded. If it is not the case, there would exist a subsequence of $\left\{q_{k}\right\}$, still denoted by $\left\{q_{k}\right\}$, such that $\lim _{k \rightarrow \infty}\left|\eta_{q_{k}} y_{q_{k}}\right|=+\infty$, then by (3.42), we have

$$
\int_{\mathbb{R}^{2}} V(x) u_{q_{k}}^{2} d x=\int_{\mathbb{R}^{2}} V\left(\eta_{q_{k}}\left(x+y_{q_{k}}\right)\right) f_{q_{k}}^{2}(x) d x \geq \int_{B_{R_{0}}(0)} V\left(\eta_{q_{k}}\left(x+y_{q_{k}}\right)\right) f_{q_{k}}^{2}(x) d x \rightarrow \infty
$$


which is a contradiction to (3.30). By (2.21), (3.32), (3.33), (3.30) and (3.38), we get

$$
\begin{aligned}
\lim _{k \rightarrow \infty} \eta_{q_{k}}^{2} \mu_{q_{k}} & =\lim _{k \rightarrow \infty} \frac{\mu_{q_{k}}}{\left\|\nabla u_{q_{k}}\right\|_{2}^{2}} \\
& =1+\lim _{k \rightarrow \infty} \frac{\int_{\mathbb{R}^{2}} V(x) u_{q_{k}}^{2} d x}{\left\|\nabla u_{q_{k}}\right\|_{2}^{2}}-\lim _{k \rightarrow \infty} \frac{a \int_{\mathbb{R}^{2}}\left|u_{q_{k}}\right|^{q_{k}+2} d x}{\left\|\nabla u_{q_{k}}\right\|_{2}^{2}} \\
& =-1 .
\end{aligned}
$$

By (3.32), (3.33), (3.39) and (3.44), letting $k \rightarrow \infty$ in (3.43), we have that $f \neq 0$ solves

$$
-\Delta f+f=a f^{3} \text {. }
$$

Using Lemma 2.3 with $V=1$, we have

$$
\int_{\mathbb{R}^{2}}|\nabla f|^{2} d x=\frac{a}{2} \int_{\mathbb{R}^{2}} f^{4} d x
$$

By the Gagliardo-Nirenberg inequality (see [17]) and $\|f\|_{2}^{2} \leq 1$, we obtain

$$
\int_{\mathbb{R}^{2}} f^{4} d x \leq \frac{2}{a^{*}} \int_{\mathbb{R}^{2}}|\nabla f|^{2} d x \int_{\mathbb{R}^{2}} f^{2} d x=\frac{a}{a^{*}} \int_{\mathbb{R}^{2}} f^{4} d x \int_{\mathbb{R}^{2}} f^{2} d x \leq \frac{a}{a^{*}} \int_{\mathbb{R}^{2}} f^{4} d x,
$$

which is a contradiction since $a<a^{*}$ and $f \neq 0$. The assertion follows.

Now, we are in position to prove Theorem 1.2.

Proof of Theorem 1.2. We first prove $(i)$.

By Lemma 3.3, there exists $u_{0} \in \mathcal{H}$ such that $u_{q} \rightarrow u_{0}$ weakly in $\mathcal{H}$, and Lemma 3.1 implies that $u_{q} \rightarrow u_{0}$ in $L^{p}\left(\mathbb{R}^{2}\right)$ for any $p \geq 2$. Now we prove $u_{q} \rightarrow u_{0}$ in $\mathcal{H}$ as $q \rightarrow 2$. Since $u_{q}$ satisfies

$$
-\Delta u_{q}+V(x) u_{q}=\mu_{q} u_{q}+a\left(u_{q}\right)^{q+1},
$$

where

$$
\mu_{q}=\int_{\mathbb{R}^{2}}\left(\left|\nabla u_{q}\right|^{2}+V(x)\left|u_{q}\right|^{2}\right) d x-a \int_{\mathbb{R}^{2}}\left|u_{q}\right|^{q+2} d x
$$

we see that $\mu_{q}$ is uniformly bounded in $q$. Suppose $\mu_{q} \rightarrow \mu_{0}$ as $q \rightarrow 2_{+}$, then $u_{0}$ satisfies

$$
-\Delta u_{0}+V(x) u_{0}=\mu_{0} u_{0}+a u_{0}^{3}
$$

implying

$$
\int_{\mathbb{R}^{2}}\left|\nabla u_{0}\right|^{2}+V(x)\left|u_{0}\right|^{2} d x=\mu_{0}+a \int_{\mathbb{R}^{2}}\left|u_{0}\right|^{4} d x .
$$

We deduce from the Brézis-Lieb lemma and

$$
\int_{\mathbb{R}^{2}}\left|\nabla u_{q}\right|^{2}+V(x)\left|u_{q}\right|^{2} d x=\mu_{q}+a \int_{\mathbb{R}^{2}}\left|u_{q}\right|^{q+2} d x
$$

that

$$
\begin{aligned}
& \int_{\mathbb{R}^{2}}\left|\nabla u_{q}-\nabla u_{0}\right|^{2} d x+\int_{\mathbb{R}^{2}} V(x)\left|u_{q}-u_{0}\right|^{2} d x \\
& =\mu_{q}-\mu_{0}+a \int_{\mathbb{R}^{2}}\left|u_{q}-u_{0}\right|^{q+2} d x+a \int_{\mathbb{R}^{2}}\left|u_{0}\right|^{q+2} d x-a \int_{\mathbb{R}^{2}}\left|u_{0}\right|^{4} d x+o(1),
\end{aligned}
$$


as $q \rightarrow 2_{+}$. This implies $u_{q} \rightarrow u_{0}$ in $\mathcal{H}$.

We claim that $u_{0}$ is a minimizer of

$$
d_{a}(2)=\inf _{u \in \mathcal{H},\|u\|_{2}=1} E_{a, 2}(u) .
$$

In fact, suppose on the contrary that

$$
E_{a, 2}\left(u_{0}\right)>d_{a}(2)=E_{a, 2}(w),
$$

where $w$ is a minimizer of $d_{a}(2)$. By the convergence of $u_{q} \rightarrow u_{0}$ in $\mathcal{H}$, for $q$ close to 2 there exists $\varepsilon_{0} \in\left(0, \frac{1}{3}\left(E_{a, 2}\left(u_{0}\right)-E_{a, 2}(w)\right)\right)$ such that

$$
E_{a, 2}(w) \geq E_{a, q}(w)-\varepsilon_{0}, \quad E_{a, 2}\left(u_{0}\right) \leq E_{a, q}\left(u_{q}\right)+\varepsilon_{0}
$$

and

$$
\left\|\nabla u_{0}\right\|_{2}^{2}+\|\nabla w\|_{2}^{2} \leq \tau_{q}^{2}
$$

As a result,

$$
E_{a, q}\left(u_{q}\right) \geq E_{a, 2}\left(u_{0}\right)-\varepsilon_{0}>E_{a, 2}(w)+\varepsilon_{0} \geq E_{a, q}(w) .
$$

It yields

$$
E_{a, q}\left(u_{q}\right)>E_{a, q}(w) \geq \inf _{u \in A_{\tau_{q}^{2}}} E_{a, q}(u),
$$

which is a contradiction to the fact $E_{a, q}\left(u_{q}\right)=\inf _{u \in A_{\tau_{q}^{2}}} E_{a, q}(u)$.

Now we prove $(i i)$, that is, $\left\{v_{q}\right\}$ is unbounded in $H^{1}\left(\mathbb{R}^{2}\right)$. Suppose by the contradiction that $\left\{v_{q}\right\}$ is bounded in $H^{1}\left(\mathbb{R}^{2}\right)$, then by the Sobolev embedding theorem, $\left\{v_{q}\right\}$ is bounded in $L^{q+2}\left(\mathbb{R}^{2}\right)$. Noting equation (3.6) implies that

$$
c_{q}=E_{a, q}\left(v_{q}\right) \geq\left.\inf _{u \in \partial A_{\tau_{q}^{2}}} E_{a, q}\right|_{V=0}(u) \geq \frac{q-2}{2 q} \tau_{q}^{2} \rightarrow+\infty
$$

as $q \rightarrow 2_{+}$, we infer that

$$
\int_{\mathbb{R}^{2}} V(x)\left|v_{q}\right|^{2} d x \rightarrow+\infty
$$

as $q \rightarrow 2_{+}$. This and (3.46) yield $\mu_{q} \rightarrow \infty$, as $q \rightarrow 2_{+}$. However, this is impossible. In fact, since $-\Delta+V$ is a compact operator, so it has a discrete spectrum, and the first eigenvalue $\lambda_{1}$ and corresponding eigenfunction $\varphi_{1}$ are positive, which satisfy

$$
-\Delta \varphi_{1}+V \varphi_{1}=\lambda_{1} \varphi_{1} .
$$

Hence, we obtain

$$
\begin{aligned}
\int_{\mathbb{R}^{2}} \mu_{q} v_{q} \varphi_{1} d x & =\int_{\mathbb{R}^{2}}\left(-\Delta v_{q}+V v_{q}-a v_{q}^{q+1}\right) \varphi_{1} d x \\
& <\int_{\mathbb{R}^{2}}\left(-\Delta \varphi_{1}+V \varphi_{1}\right) v_{q} d x \\
& =\int_{\mathbb{R}^{2}} \lambda_{1} \varphi_{1} v_{q} d x
\end{aligned}
$$

That is

$$
\mu_{q}<\lambda_{1},
$$


which is a contradiction.

Finally, we show that the assumption $\left(V_{e}\right)$ implies $\left(V_{1}\right)$ and $\left(V_{2}\right)$, that is, the result of Corollary 1.1. Therefore, the consequences in Theorem 1.1 hold true if we assume $\left(V_{e}\right)$.

Proof of Corollary 1.1 We will verify that $V$ satisfies condition $\left(V_{1}\right)$ and $\left(V_{2}\right)$. Obviously, $\left(V_{1}\right)$ holds true. So we need only to verify $\left(V_{2}\right)$. It holds that

$$
x \cdot \nabla V=\sum_{i=1}^{n} p_{i} V+\sum_{i=1}^{n} p_{i} x_{i} \prod_{m \neq i}\left|x-x_{m}\right|^{p_{m}}\left|x-x_{i}\right|^{p_{i}-2}\left(x-x_{i}\right) .
$$

Let

$$
f_{i}=p_{i} x_{i} \prod_{m \neq i}\left|x-x_{m}\right|^{p_{m}}\left|x-x_{i}\right|^{p_{i}-2}\left(x-x_{i}\right) .
$$

Choose $\delta_{i}>0$ such that

$$
\sum_{i=1}^{n}\left|p_{i} x_{i} \delta_{i}\right|<\frac{p}{2}
$$

If $\left|x-x_{i}\right| \geq \frac{1}{\delta_{i}}$, we have $\left|f_{i}\right| \leq\left|p_{i} x_{i} \delta_{i}\right| V$; and if $\left|x-x_{i}\right| \leq \frac{1}{\delta_{i}}$, then $\left|f_{i}\right| \leq C_{i}$ for some $C_{i}>0$. Hence, there exists $C>0$ such that

$$
|x \cdot \nabla V(x)| \leq C(V(x)+1)
$$

and

$$
x \cdot \nabla V \geq \sum_{i=1}^{n} p_{i} V-\sum_{i=1}^{n}\left(\left|p_{i} x_{i} \delta_{i}\right| V+C_{i}\right) \geq \frac{p}{2} V-\sum_{i=1}^{n} C_{i} .
$$

Thus, $\left(V_{2}\right)$ immediately follows.

\section{Energy estimates}

It is known from Theorem 1.2 that the local minimizer $u_{q}$ of $E_{a, q}$ tends to a minimizer $u_{0} \in H^{1}\left(\mathbb{R}^{2}\right)$ of $d_{a}(2)$. Let $v_{q}$ be the mountain pass point of $E_{a, q}$ obtained by Corollary 1.1. In this section, we focus on investigating the asymptotic behavior of energy $E_{a, q}\left(v_{q}\right)$ as $q \rightarrow 2_{+}$. We suppose in this and next sections that $V$ satisfies condition $\left(V_{e}\right)$.

Proposition 4.1. There holds

$$
E_{a, q}\left(v_{q}\right)=\frac{q-2}{2 q}\left(\frac{2 a_{q}^{*}}{q a}\right)^{\frac{2}{q-2}}+o(1)
$$

as $q \rightarrow 2_{+}$. 
Proof. It follows from (3.6) and the definition of $c_{q}$ that

$$
c_{q}=E_{a, q}\left(v_{q}\right) \geq \inf _{u \in \partial A_{\tau_{q}^{2}}} \tilde{E}_{a, q}\left(v_{q}\right) \geq \frac{q-2}{2 q}\left(\frac{2 a_{q}^{*}}{q a}\right)^{\frac{2}{q-2}} .
$$

Now, we prove

$$
E_{a, q}\left(v_{q}\right) \leq \frac{q-2}{2 q}\left(\frac{2 a_{q}^{*}}{q a}\right)^{\frac{2}{q-2}}+o(1)
$$

as $q \rightarrow 2_{+}$. To this purpose, we will construct a path $g$ in $\Gamma_{q}$ defined in (3.22) connecting $\varphi^{t_{0}}$ and $\varphi^{t_{1}}$ so that

$$
E_{a, q}\left(v_{q}\right) \leq \max _{t \geq 0} E_{a, q}(g(t)) \leq \frac{q-2}{2 q}\left(\frac{2 a_{q}^{*}}{q a}\right)^{\frac{2}{q-2}}+o(1) .
$$

The path $g$ is constructed in three parts. First, we construct a path $g_{1}$ connecting $\varphi^{t_{0}}$ to some $w_{q}^{\tilde{t}_{0}}$, and estimate $E_{a, q}\left(g_{1}(s)\right)$.

Let $\lambda_{i} \in(0, \infty]$ be given by

$$
\lambda_{i}=\lim _{x \rightarrow x_{i}} \frac{V(x)}{\left|x-x_{i}\right|^{p_{\max }}} .
$$

Define $\lambda=\min \left\{\lambda_{1}, \cdots, \lambda_{n}\right\}$, and denote $\mathcal{Z}:=\left\{x_{i} \mid \lambda_{i}=\lambda\right\}$ and $p=\sum_{i=1}^{n} p_{i}$. Let

$$
w_{q}(x)=\frac{\tau_{q} \varphi_{q}\left(\tau_{q}\left(x-x_{0}\right)\right)}{\left\|\varphi_{q}\right\|_{2}},
$$

where $\varphi_{q}$ is the unique positive solution of (1.8) and $x_{0} \in \mathcal{Z}$. Then,

$$
\int_{\mathbb{R}^{2}}\left|w_{q}\right|^{2} d x=1
$$

By (2.1) and (2.4), we deduce that

$$
\int_{\mathbb{R}^{2}}\left|\nabla w_{q}\right|^{2} d x=\tau_{q}^{2} \frac{\int_{\mathbb{R}^{2}}\left|\nabla \varphi_{q}\right|^{2} d x}{\int_{\mathbb{R}^{2}}\left|\varphi_{q}\right|^{2} d x}=\tau_{q}^{2},
$$

and

$$
\int_{\mathbb{R}^{2}}\left|w_{q}\right|^{q+2} d x=\tau_{q}^{q} \frac{\int_{\mathbb{R}^{2}}\left|\varphi_{q}\right|^{q+2} d x}{\left\|\varphi_{q}\right\|_{2}^{q+2}}=\frac{q+2}{2 a_{q}^{*}} \tau_{q}^{q} .
$$

Let

and

$$
\tilde{t}_{0}^{2}=\frac{1}{6} \theta:=\frac{1}{6} \frac{q-2}{2 q}
$$

By (4.3) we have

$$
w_{q}^{\tilde{t}_{0}}(x)=\tilde{t}_{0} w_{q}\left(\tilde{t}_{0} x\right)
$$

$$
\int_{\mathbb{R}^{2}}\left|\nabla w_{q}^{\tilde{t}_{0}}\right|^{2} d x=\tilde{t}_{0}^{2} \int_{\mathbb{R}^{2}}\left|\nabla w_{q}\right|^{2} d x=\frac{1}{6} \theta \tau_{q}^{2} .
$$

Define a path $g_{1}$ connecting $\left(w_{q}\right)^{\tilde{t_{0}}}$ and $\varphi^{t_{0}}$ as follows.

$$
g_{1}(s):=\frac{s w_{q}^{\tilde{t}_{0}}+(1-s) \varphi^{t_{0}}}{\left\|s w_{q}^{\tilde{t}_{0}}+(1-s) \varphi^{t_{0}}\right\|_{2}}, \quad s \in[0,1] .
$$


We may verify that

$$
\begin{aligned}
\left\|s w_{q}^{\tilde{t}_{0}}+(1-s) \varphi^{t_{0}}\right\|_{2}^{2} & \geq s^{2}\left\|w_{q}^{\tilde{t}_{0}}\right\|_{2}^{2}+(1-s)^{2}\left\|\varphi^{t_{0}}\right\|_{2}^{2} \\
& =s^{2}+(1-s)^{2} \geq \frac{1}{2} .
\end{aligned}
$$

By (3.9) and (4.7), we have

$$
\begin{aligned}
& \left\|s \nabla w_{q}^{\tilde{t}_{0}}+(1-s) \nabla \varphi^{t_{0}}\right\|_{2}^{2} \\
\leq & 2\left(s^{2}\left\|\nabla w_{q}^{\tilde{t}_{0}}\right\|_{2}^{2}+(1-s)^{2}\left\|\nabla \varphi^{t_{0}}\right\|_{2}^{2}\right) \\
\leq & 2\left(\left\|\nabla w_{q}^{\tilde{t}_{0}}\right\|_{2}^{2}+\left\|\nabla \varphi^{t_{0}}\right\|_{2}^{2}\right) \\
= & 2\left(\frac{1}{6} \theta \tau_{q}^{2}+\frac{1}{4} \theta \tau_{q}^{2}\right)=\frac{5}{6} \theta \tau_{q}^{2} .
\end{aligned}
$$

It follows from (4.8) and (4.9) that

$$
\left.E_{a, q}\right|_{V=0}\left(g_{1}(s)\right) \leq \frac{1}{2}\left\|\nabla g_{1}(s)\right\|_{2}^{2} \leq \frac{5}{6} \theta \tau_{q}^{2} .
$$

On the other hand, by (4.8),

$$
\begin{aligned}
\int_{\mathbb{R}^{2}} V(x)\left|g_{1}(s)\right|^{2} d x & \leq 2 \int_{\mathbb{R}^{2}} V(x)\left|s w_{q}^{\tilde{t}_{0}}+(1-s) \varphi^{t_{0}}\right|^{2} d x \\
& \leq 4\left(\int_{\mathbb{R}^{2}} V(x)\left|w_{q}^{\tilde{t}_{0}}\right|^{2} d x+\int_{\mathbb{R}^{2}} V(x)\left|\varphi^{t_{0}}\right|^{2} d x\right) .
\end{aligned}
$$

Recall

we claim that

$$
V\left(\frac{x}{t}\right)=\left|\frac{x}{t}-x_{1}\right|^{p_{1}}\left|\frac{x}{t}-x_{2}\right|^{p_{2}} \cdots\left|\frac{x}{t}-x_{n}\right|^{p_{n}}
$$

$$
V\left(\frac{x}{t}\right) \leq C t^{-\sum_{i=1}^{n} p_{i}}|x|^{\sum_{i=1}^{n} p_{i}}+C=C t^{-p}|x|^{p}+C,
$$

where $C>0$ is independent of $q$. Indeed, if $\left|\frac{x}{t}\right| \geq \max _{i}\left|x_{i}\right|$, then

$$
\left|\frac{x}{t}-x_{i}\right| \leq\left|\frac{x}{t}\right|+\left|x_{i}\right| \leq 2\left|\frac{x}{t}\right|
$$

implies

$$
V\left(\frac{x}{t}\right) \leq 2^{p}\left|\frac{x}{t}\right|^{p}
$$

If $\left|\frac{x}{t}\right| \leq \max _{i}\left|x_{i}\right|$, we have

$$
\left|\frac{x}{t}-x_{i}\right| \leq\left|\frac{x}{t}\right|+\left|x_{i}\right| \leq 2 \max _{i}\left|x_{i}\right|
$$

and then

$$
V\left(\frac{x}{t}\right) \leq\left(2 \max \left|x_{i}\right|\right)^{p} .
$$

Consequently,

$$
V\left(\frac{x}{t}\right) \leq 2^{p}\left|\frac{x}{t}\right|^{p}+\left(2 \max \left|x_{i}\right|\right)^{p},
$$


that is, the claim is valid. By (4.2) and (4.12), we deduce

$$
\begin{aligned}
& \int_{\mathbb{R}^{2}} V(x)\left|w_{q}^{\tilde{t}_{0}}\right|^{2} d x \\
& =\int_{\mathbb{R}^{2}} V\left(\frac{x}{\tilde{t}_{0}}\right)\left|w_{q}\right|^{2} d x \\
& \leq C \int_{\mathbb{R}^{2}} \tilde{t}_{0}^{-p}|x|^{p}\left|w_{q}\right|^{2} d x+\tilde{C} \\
& =\frac{\tilde{t}_{0}^{-p}}{\left\|\varphi_{q}\right\|_{2}^{2}} \int_{\mathbb{R}^{2}}\left|\frac{x}{\tau_{q}}+x_{0}\right|^{p} \varphi_{q}^{2}(x) d x+\tilde{C} .
\end{aligned}
$$

By (2.3) and the Lebesgue dominated theorem, we get

$$
\int_{\mathbb{R}^{2}}\left|\frac{x}{\tau_{q}}+x_{0}\right|^{p} \frac{\left|\varphi_{q}(x)\right|^{2}}{\left\|\varphi_{q}\right\|_{2}^{2}} d x \rightarrow\left|x_{0}\right|^{p}
$$

since $\tau_{q} \rightarrow \infty$ as $q \rightarrow 2_{+}$. It follows from (4.13) and (4.14) that for $q \rightarrow 2_{+}$,

$$
\int_{\mathbb{R}^{2}} V(x)\left|w_{q}^{\tilde{t}_{0}}\right|^{2} d x \leq O\left(\tilde{t}_{0}^{-p}\right) .
$$

By (3.10), (4.11) and (4.15), we have

$$
\int_{\mathbb{R}^{2}} V(x)\left|g_{1}(s)\right|^{2} d x \leq O\left(\tilde{t}_{0}^{-p}\right)+8 V(0) .
$$

Taking into account (4.5), (4.10) and (4.16), we obtain

$$
\begin{aligned}
E_{a, q}\left(g_{1}(s)\right) & \leq \frac{5}{6} \theta \tau_{q}^{2}+O\left(\tilde{t}_{0}^{-p}\right)+8 V(0) \\
& =\frac{5}{6} \frac{q-2}{2 q}\left(\frac{2 a_{q}^{*}}{q a}\right)^{\frac{2}{q-2}}+O\left((q-2)^{-\frac{p}{2}}\right)+8 V(0) .
\end{aligned}
$$

Next, we construct the second part of the path $g$. Let

$$
\tilde{t}_{1}=\tau_{q}^{-1} t_{1}
$$

and

$$
w_{q}^{\tilde{t}_{1}}=\tilde{t}_{1} w_{q}\left(\tilde{t}_{1} x\right)
$$

where $t_{1}$ is defined in (3.21) and $w_{q}$ is defined in (4.1). We define a path $g_{2}$ connecting $w_{q}^{\tilde{t}_{1}}$ and $\varphi^{t_{1}}$ as follows.

$$
g_{2}(s)=\frac{s w_{q}^{\tilde{t}_{1}}+(1-s) \varphi^{t_{1}}}{\left\|s w_{q}^{\tilde{t}_{1}}+(1-s) \varphi^{t_{1}}\right\|_{2}}, \quad s \in[0,1] .
$$

By (4.3) and (4.8), we get

$$
\begin{aligned}
\left\|\nabla g_{2}(s)\right\|_{2}^{2} & \leq 2\left\|s \nabla w_{q}^{\tilde{t}_{1}}+(1-s) \nabla \varphi^{t_{1}}\right\|_{2} \\
& \leq 4\left(s^{2}\left\|\nabla w_{q}^{\tilde{t}_{1}}\right\|_{2}^{2}+(1-s)^{2}\left\|\nabla \varphi^{t_{1}}\right\|_{2}^{2}\right. \\
& =4\left(s^{2} \tilde{t}_{1}^{2} \tau_{q}^{2}+(1-s)^{2} t_{1}^{2}\|\nabla \varphi\|_{2}^{2}\right) .
\end{aligned}
$$


Since $\left\|w_{q}^{\tilde{t}_{1}}\right\|_{2}^{2}=\left\|\varphi^{t_{1}}\right\|_{2}^{2}=1$, we have

$$
\left\|s w_{q}^{\tilde{t}_{1}}+(1-s) \varphi^{t_{1}}\right\|_{2}^{2} \leq 2\left[\left\|s w_{q}^{\tilde{t}_{1}}\right\|_{2}^{2}+\left\|(1-s) \varphi^{t_{1}}\right\|_{2}^{2}\right] \leq 4 .
$$

This with (4.4) yields that

$$
\begin{aligned}
\int_{\mathbb{R}^{2}}\left|g_{2}(s)\right|^{q+2} d x & \geq 2^{-(q+2)} \int_{\mathbb{R}^{2}}\left|s w_{q}^{\tilde{t}_{1}}+(1-s) \varphi^{t_{1}}\right|^{q+2} d x \\
& \geq 2^{-q-3}\left(s^{q+2} \int_{\mathbb{R}^{2}}\left|w_{q}^{\tilde{t}_{1}}\right|^{q+2} d x+(1-s)^{q+2} \int_{\mathbb{R}^{2}}\left|\varphi^{t_{1}}\right|^{q+2} d x\right) \\
& =2^{-q-3}\left(\frac{q+2}{2 a_{q}^{*}} s^{q+2} \tilde{t}_{1}^{q} \tau_{q}^{q}+(1-s)^{q+2} t_{1}^{q} \int_{\mathbb{R}^{2}}|\varphi|^{q+2} d x\right) .
\end{aligned}
$$

Therefore,

$$
\begin{aligned}
\left.E_{a, q}\right|_{V=0}\left(g_{2}(s)\right) & \leq 2\left(s^{2} \tilde{t}_{1}^{2} \tau_{q}^{2}+(1-s)^{2} t_{1}^{2}\|\nabla \varphi\|_{2}^{2}\right) \\
& -\frac{a}{2^{q+3}(q+2)}\left(\frac{q+2}{2 a_{q}^{*}} s^{q+2} \tilde{t}_{1}^{q} \tau_{q}^{q}+(1-s)^{q+2} t_{1}^{q} \int_{\mathbb{R}^{2}}|\varphi|^{q+2} d x\right) .
\end{aligned}
$$

If $0<s \leq \frac{1}{2}$, we have

$$
\begin{aligned}
\left.E_{a, q}\right|_{V=0}\left(g_{2}(s)\right) & \leq 2 \tilde{t}_{1}^{2} \tau_{q}^{2}+2 t_{1}^{2}\|\nabla \varphi\|_{2}^{2}-\frac{a}{2^{q+3}(q+2)}(1-s)^{q+2} t_{1}^{q} \int_{\mathbb{R}^{2}}|\varphi|^{q+2} d x \\
& \leq 2 t_{1}^{2}+2 t_{1}^{2}\|\nabla \varphi\|_{2}^{2}-\frac{a}{2^{2 q+5}(q+2)} t_{1}^{q} \int_{\mathbb{R}^{2}}|\varphi|^{q+2} d x .
\end{aligned}
$$

Since $t_{1}^{q} / t_{1}^{2}=2^{\frac{1}{q-2}} C^{q-2} \rightarrow \infty$ as $q \rightarrow 2+$, by (3.21) we find for $q$ close to 2 that

$$
\left.E_{a, q}\right|_{V=0}\left(g_{2}(s)\right) \leq-\frac{a C}{2^{2 q+6} q+2} 2^{\frac{q}{(q-2)^{2}}} \int_{\mathbb{R}^{2}}|\varphi|^{q+2} d x .
$$

If $\frac{1}{2} \leq s \leq 1$, we deduce in the same way that for $q$ close to 2

$$
\begin{aligned}
\left.E_{a, q}\right|_{V=0}\left(g_{2}(s)\right) & \leq 2\left(s^{2} \widetilde{t}_{1}^{2} \tau_{q}^{2}+(1-s)^{2} t_{1}^{2}\|\nabla \varphi\|_{2}^{2}\right)-\frac{a}{2^{q+3}(q+2)} \frac{q+2}{2 a_{q}^{*}} s^{q+2} \tilde{t}_{1}^{q} \tau_{q}^{q} \\
& \leq 2\left(t_{1}^{2}+t_{1}^{2}\|\nabla \varphi\|_{2}^{2}\right)-\frac{a}{2^{2 q+5}(q+2)} \frac{q+2}{2 a_{q}^{*}} t_{1}^{q} \\
& \leq-\frac{a}{2^{2 q+6}(q+2)} \frac{q+2}{2 a_{q}^{*}} t_{1}^{q} .
\end{aligned}
$$

Thus,

$$
\left.E_{a, q}\right|_{V=0}\left(g_{2}(s)\right) \leq-C t_{1}^{q}=-2^{\frac{q-1}{(q-2)^{2}}} C .
$$

We may prove as (4.15) that

Consequently,

$$
\int_{\mathbb{R}^{2}} V(x)\left|g_{2}(s)\right|^{2} d x \leq C .
$$

$$
E_{a, q}\left(g_{2}(s)\right)<0
$$

for $q>2$ and close to 2 . 
Finally, we construct a path linking $w_{q}^{\tilde{t}_{0}}$ and $w_{q}^{\tilde{t}_{1}}$.

Since $(4.7)$ and $\left\|\nabla w_{q}^{\tilde{t}_{1}}\right\|_{2}^{2}=2^{\frac{2}{(q-2)^{2}}} C$, there exists a $\tilde{t}_{2} \in\left(\tilde{t}_{0}, \tilde{t}_{1}\right)$ such that $\left\|\nabla w_{q}^{\tilde{t}_{2}}\right\|=\tau_{q}^{2}$. By (3.6), we have

$$
E_{a, q}\left(w_{q}^{\tilde{t}_{2}}\right) \geq\left. E_{a, q}\right|_{V=0}\left(w_{q}^{\tilde{t}_{2}}\right) \geq \theta \tau_{q}^{2} .
$$

Define a path linking $w_{q}^{\tilde{t}_{0}}$ and $w_{q}^{\tilde{t}_{1}}$ as follows.

$$
g_{3}(s)=w_{q}^{s \tilde{t}_{0}+(1-s) \tilde{t}_{1}}, \quad s \in[0,1] .
$$

Now, we define a path $g$ in $\Gamma_{q}$ defined in (3.20) by $g_{1}, g_{2}$ and $g_{3}$. Precisely, we define $g(s)=g_{1}(3 s)$ if $0 \leq s \leq \frac{1}{3} ; g(s)=g_{3}(3 s-1)$ if $\frac{1}{3} \leq s \leq \frac{2}{3}$, and $g(s)=g_{2}(3 s-2)$ if $\frac{2}{3} \leq s \leq 1$. Then, $g(s) \in \Gamma_{q}$.

By (4.17), (4.23) and (4.24), we have

$$
\max _{\{0 \leq s \leq 1\}} E_{a, q}(g(s))=\max _{\left\{\frac{1}{3} \leq s \leq \frac{2}{3}\right\}} E_{a, q}\left(g_{3}(3 s-1)\right)=\max _{\left\{\tilde{t}_{0} \leq t \leq \tilde{t}_{1}\right\}} E_{a, q}\left(w_{q}^{t}\right) .
$$

Apparently, by (4.3) and (4.4), there exists $t_{1, q} \in\left(\tilde{t}_{0}, \tilde{t}_{1}\right)$ such that

$$
E_{a, q}\left(w_{q}^{t_{1, q}}\right)=\max _{\left\{\tilde{t}_{0} \leq t \leq \tilde{t}_{1}\right\}} E_{a, q}\left(w_{q}^{t}\right)=: \max _{\left\{\tilde{t}_{0} \leq t \leq \tilde{t}_{1}\right\}} f(t)
$$

with $f^{\prime}\left(t_{1, q}\right)=0$, where

$$
f(t)=\frac{1}{2} t^{2} \tau_{q}^{2}-\frac{1}{q} t^{q} \tau_{q}^{2}+\frac{1}{2} \int_{\mathbb{R}^{2}} V\left(\frac{x}{t}\right)\left|w_{q}\right|^{2} d x .
$$

and

$$
f^{\prime}(t)=t \tau_{q}^{2}-t^{q-1} \tau_{q}^{2}-\frac{1}{2 t^{2}} \int_{\mathbb{R}^{2}} x \cdot \nabla V\left(\frac{x}{t}\right)\left|w_{q}\right|^{2} d x
$$

Similar to the proof of (4.12), we have

$$
\left|\nabla V\left(\frac{x}{t}\right)\right|=\sum_{1}^{n} p_{i}\left|\frac{x}{t}-x_{i}\right|^{p_{i}-1} \Pi_{m \neq i}\left|\frac{x}{t}-x_{m}\right|^{p_{m}} \leq C t^{-p+1}|x|^{p-1}+C .
$$

Therefore,

$$
\begin{aligned}
& \left.\left|\int_{\mathbb{R}^{2}} x \cdot \nabla V\left(\frac{x}{t}\right)\right| w_{q}\right|^{2} d x \mid \\
& \leq \int_{\mathbb{R}^{2}} C t^{-p+1}|x|^{p}\left|w_{q}\right|^{2} d x+\int_{\mathbb{R}^{2}} C|x|\left|w_{q}\right|^{2} d x \\
& =\int_{\mathbb{R}^{2}} C t^{-p+1}|x|^{p} \tau_{q}^{2}\left|\varphi_{q}\left(\tau_{q}\left(x-x_{0}\right)\right)\right|^{2} d x+\int_{\mathbb{R}^{2}} C|x| \tau_{q}^{2}\left|\varphi_{q}\left(\tau_{q}\left(x-x_{0}\right)\right)\right|^{2} d x .
\end{aligned}
$$

By the change of variables, we estimate

$$
\begin{aligned}
& \int_{\mathbb{R}^{2}} t^{-p+1}|x|^{p} \tau_{q}^{2}\left|\varphi_{q}\left(\tau_{q}\left(x-x_{0}\right)\right)\right|^{2} d x \\
& \leq C \int_{\mathbb{R}^{2}} t^{-p+1}|x|^{p} \tau_{q}^{-p}\left|\varphi_{q}(x)\right|^{2} d x+C t^{-p+1}\left|x_{0}\right|^{p} \int_{\mathbb{R}^{2}}\left|\varphi_{q}\right|^{2} d x .
\end{aligned}
$$


We remark that the integral $\int_{\mathbb{R}^{2}}|x|^{p}\left|\varphi_{q}(x)\right|^{2} d x$ is finite because $\varphi_{q}$ exponentially decays at infinity uniformly in $q$. Therefore,

$$
\int_{\mathbb{R}^{2}} t^{-p+1}|x|^{p} \tau_{q}^{2}\left|\varphi_{q}\left(\tau_{q}\left(x-x_{0}\right)\right)\right|^{2} d x \leq C t^{-p+1} \tau_{q}^{-p}+C t^{-p+1} \leq C t^{-p+1} .
$$

Similarly, we have

$$
\int_{\mathbb{R}^{2}}|x| \tau_{q}^{2}\left|\varphi_{q}\left(\tau_{q}\left(x-x_{0}\right)\right)\right|^{2} d x \leq C\left(\tau_{q}^{-1}+\left|x_{0}\right|\right) \leq C
$$

Hence, if $t \geq \tilde{t}_{0}=\frac{q-2}{12 q}$, we have

$$
\left.\frac{1}{2 t^{2}}\left|\int_{\mathbb{R}^{2}} x \cdot \nabla V\left(\frac{x}{t}\right)\right| w_{q}\right|^{2} d x \mid \leq C t^{-p-1}+C t^{-2} \leq C\left(\frac{q-2}{12 q}\right)^{\min \{-p-1,-2\}} .
$$

This allows us to infer from $f^{\prime}\left(t_{1, q}\right)=0$ that

$$
\left|t_{1, q} \tau_{q}^{2}-t_{1, q}^{q-1} \tau_{q}^{2}\right| \leq C\left(\frac{q-2}{12 q}\right)^{\min \{-p-1,-2\}} .
$$

We claim that $t_{1, q} \rightarrow 1$ as $q \rightarrow 2_{+}$. Indeed, were it not the case, there would exist $\varepsilon_{0}>0$ small and $q_{n} \rightarrow 2_{+}$such that either $t_{1, q_{n}} \geq 1+\varepsilon_{0}$ or $t_{1, q_{n}} \leq 1-\varepsilon_{0}$.

If $t_{1, q_{n}} \geq 1+\varepsilon_{0}$, noting

$$
\lim _{t \rightarrow 0_{+}} \frac{1-\left(1+\varepsilon_{0}\right)^{t}}{t}=-\ln \left(1+\varepsilon_{0}\right)
$$

we obtain

$$
\begin{aligned}
& t_{1, q_{n}} \tau_{q_{n}}^{2}-t_{1, q_{n}}^{q_{n}-1} \tau_{q_{n}}^{2} \\
\leq & t_{1, q_{n}} \tau_{q_{n}}^{2}\left[1-\left(1+\varepsilon_{0}\right)^{q_{n}-2}\right] \\
\leq & -\frac{1}{2} t_{1, q_{n}} \tau_{q_{n}}^{2}\left(q_{n}-2\right) \ln \left(1+\varepsilon_{0}\right) \\
\leq & -\frac{1}{2}\left(1+\varepsilon_{0}\right) \ln \left(1+\varepsilon_{0}\right)\left(q_{n}-2\right) \tau_{q_{n}}^{2} .
\end{aligned}
$$

This contradicts $(4.28)$ since

$$
\lim _{n \rightarrow \infty} \frac{\left(q_{n}-2\right) \tau_{q_{n}}^{2}}{\left(\frac{q_{n}-2}{12 q_{n}}\right)^{\min \{-p-1,-2\}}}=+\infty .
$$

Observe $\tilde{t}_{1}>t_{1, q}>\tilde{t}_{0}=\frac{1}{6} \frac{q-2}{2 q}$, if $t_{1, q_{n}} \leq 1-\varepsilon_{0}$, using the fact that

$$
\lim _{t \rightarrow 0_{+}} \frac{1-\left(1-\varepsilon_{0}\right)^{t}}{t}=-\ln \left(1-\varepsilon_{0}\right),
$$


we find

$$
\begin{aligned}
& t_{1, q_{n}} \tau_{q_{n}}^{2}-t_{1, q_{n}}^{q_{n}-1} \tau_{q_{n}}^{2} \\
& \geq t_{1, q_{n}} \tau_{q_{n}}^{2}\left[1-\left(1-\varepsilon_{0}\right)^{q_{n}-2}\right] \\
& \geq-\frac{1}{2} t_{1, q_{n}} \tau_{q_{n}}^{2}\left(q_{n}-2\right) \ln \left(1-\varepsilon_{0}\right) \\
& \geq-\frac{1}{12} \frac{q_{n}-2}{2 q_{n}} \tau_{q_{n}}^{2}\left(q_{n}-2\right) \ln \left(1-\varepsilon_{0}\right) \\
& =-\frac{\left(q_{n}-2\right)^{2}}{24 q_{n}} \ln \left(1-\varepsilon_{0}\right) \tau_{q_{n}}^{2} \rightarrow+\infty,
\end{aligned}
$$

which contradicts (4.28). Consequently, $t_{1, q} \rightarrow 1$ if $q \rightarrow 2$.

Next, we prove further that

$$
\left|1-t_{1, q}\right| \leq \tau_{q}^{-\frac{3}{2}}
$$

Since $t_{1, q} \rightarrow 1$ as $q \rightarrow 2$, we show as (4.27) that

$$
\left.\frac{1}{2 t_{1, q}^{2}}\left|\int_{\mathbb{R}^{2}} x \cdot \nabla V\left(\frac{x}{t_{1, q}}\right)\right| w_{q}\right|^{2} d x \mid \leq C t_{1, q}^{-p-1}+C t_{1, q}^{-2} \leq C .
$$

This together with $f^{\prime}\left(t_{1, q}\right)=0$ yields

$$
\left|t_{1, q} \tau_{q}^{2}-t_{1, q}^{q-1} \tau_{q}^{2}\right| \leq C,
$$

that is,

$$
\left|\left(1-t_{1, q}^{q-2}\right) \tau_{q}^{2}\right| \leq C
$$

By (3.9), (4.3) and (4.4), we have

$$
\left.E_{a, q}\right|_{V=0}\left(w_{1}^{t_{1, q}}\right)=\frac{1}{2} t_{1, q}^{2} \tau_{q}^{2}-\frac{1}{q} t_{1, q}^{q} \tau_{q}^{2} \leq \frac{q-2}{2 q} \tau_{q}^{2} .
$$

Now we are ready to prove (4.29). Suppose on the contrary that (4.29) does not hold, there would exist $t_{1, q_{n}} \rightarrow 2_{+}$such that either $t_{1, q_{n}} \leq 1-\tau_{q_{n}}^{-\frac{3}{2}}$ or $t_{1, q_{n}} \geq 1+\tau_{q_{n}}^{-\frac{3}{2}}$.

If $t_{1, q_{n}} \leq 1-\tau_{q_{n}}^{-\frac{3}{2}}$, then

$$
\left|\left(1-t_{1, q_{n}}^{q_{n}-2}\right) \tau_{q_{n}}^{2}\right|=\left(1-t_{1, q_{n}}^{q_{n}-2}\right) \tau_{q_{n}}^{2} \geq\left(1-\left(1-\tau_{q_{n}}^{-\frac{3}{2}}\right)^{q_{n}-2}\right) \tau_{q_{n}}^{2} .
$$

The limit

$$
\lim _{n \rightarrow \infty} \frac{\left(q_{n}-2\right) \ln \left(1-\tau_{q_{n}}^{-\frac{3}{2}}\right)}{\ln \left(1-\frac{1}{2}\left(q_{n}-2\right) \tau_{q_{n}}^{-\frac{3}{2}}\right)}=2
$$

implies

$$
\left(q_{n}-2\right) \ln \left(1-\tau_{q_{n}}^{-\frac{3}{2}}\right) \leq \ln \left(1-\frac{1}{2}\left(q_{n}-2\right) \tau_{q_{n}}^{-\frac{3}{2}}\right)
$$

for $n$ large, namely,

$$
\left(1-\tau_{q_{n}}^{-\frac{3}{2}}\right)^{q_{n}-2} \leq 1-\frac{1}{2}\left(q_{n}-2\right) \tau_{q_{n}}^{-\frac{3}{2}}
$$


Hence, inequality (4.32) yields that

$$
\left|\left(1-t_{1, q_{n}}^{q_{n}-2}\right) \tau_{q_{n}}^{2}\right| \geq\left[1-\left(1-2^{-1}\left(q_{n}-2\right) \tau_{q_{n}}^{-\frac{3}{2}}\right)\right] \tau_{q_{n}}^{2}=2^{-1}\left(q_{n}-2\right) \tau_{q_{n}}^{\frac{1}{2}} \rightarrow \infty,
$$

which is a contradiction to (4.30). Similarly, the case $t_{q_{n}} \geq 1+\tau_{q_{n}}^{-\frac{3}{2}}$ can also be ruled out.

Therefore, (4.29) holds true, which implies

$$
\left|\frac{x_{0} \tau_{q}}{t_{1, q}}-\tau_{q} x_{0}\right|=\left|\tau_{q} x_{0}\right| \frac{\left|1-t_{1, q}\right|}{t_{1, q}} \leq C \tau_{q}^{-\frac{1}{2}} \rightarrow 0 .
$$

We then deduce by Lemma 2.1 that

$$
\begin{aligned}
& \int_{\mathbb{R}^{2}} V(x)\left|w_{q}^{t_{1, q}}\right|^{2} d x \\
& =\int_{\mathbb{R}^{2}} V\left(\frac{x}{t_{1, q} \tau_{q}}+\frac{x_{0}}{t_{1, q}}\right) \frac{\left|\varphi_{q}(x)\right|^{2}}{\left\|\varphi_{q}\right\|_{2}^{2}} d x \\
& =\tau_{q}^{-p_{\max }} \int_{\mathbb{R}^{2}} \frac{V\left(\frac{x}{t_{1, q} \tau_{q}}+\frac{x_{0}}{t_{1, q}}\right)}{\left|\frac{x}{t_{1, q} \tau_{q}}+\frac{x_{0}}{t_{1, q}}-x_{0}\right|^{p_{\max }}}\left|\frac{x}{t_{1, q}}+\frac{x_{0} \tau_{q}}{t_{1, q}}-\tau_{q} x_{0}\right|^{p_{\max }} \frac{\left|\varphi_{q}(x)\right|^{2}}{\left\|\varphi_{q}\right\|_{2}^{2}} d x \\
& =\tau_{q}^{-p_{\max }}\|Q\|_{2}^{-2}\left(\lambda \int_{\mathbb{R}^{2}}|x|^{p_{\max }}|Q(x)|^{2} d x+o(1)\right) \\
& =\left[\lambda e^{\frac{p_{\max }}{2}}\|Q\|_{2}^{-2} \int_{\mathbb{R}^{2}}|x|^{p_{\max }}|Q(x)|^{2} d x+o(1)\right]\left(\frac{a_{q}^{*}}{a}\right)^{-\frac{p_{\max }}{q-2}}
\end{aligned}
$$

as $q \rightarrow 2$. We conclude by (4.25), (4.26), (4.31) and (4.34) that

$$
\max _{0 \leq s \leq 1} E_{a, q}(g(s)) \leq \frac{q-2}{2 q} \tau_{q}^{2}+\frac{1}{2}\left[\lambda e^{\frac{p_{\max }}{2}}\|Q\|_{2}^{-2} \int_{\mathbb{R}^{2}}|x|^{p_{\max }}|Q(x)|^{2} d x+o(1)\right]\left(\frac{a_{q}^{*}}{a}\right)^{-\frac{p_{\max }}{q-2}} .
$$

By the definition of $c_{q}$,

$$
E_{a, q}\left(v_{q}\right)=c_{q} \leq \max _{0 \leq s \leq 1} E_{a, q}(g(s))=\frac{q-2}{2 q} \tau_{q}^{2}+o(1)
$$

as $q \rightarrow 2+$. This ends the proof.

Remark 4.1. Let

$$
\tilde{c}_{q}=\frac{q-2}{2 q} \tau_{q}^{2} .
$$

It follows from (4.35) and the definition of $c_{q}$ that

$$
\limsup _{q \rightarrow 2_{+}} \frac{c_{q}-\tilde{c}_{q}}{\left(\frac{a_{q}^{*}}{a}\right)^{-\frac{p_{\max }}{q-2}}} \leq \frac{1}{2} \lambda e^{\frac{p_{\max }}{2}} \int_{\mathbb{R}^{2}} \frac{|x|^{p_{\max }}|Q(x)|^{2}}{\|Q\|_{2}^{2}} d x .
$$

Now, we estimate the gradient of $v_{q}$. 
Proposition 4.2. There exists a positive constant $C>0$ such that

$$
C(q-2) \tau_{q}^{2} \leq \int_{\mathbb{R}^{2}}\left|\nabla v_{q}\right|^{2} d x \leq \tau_{q}^{2}+\frac{C}{q-2},
$$

as $q \rightarrow 2_{+}$.

Proof. Since there exists $\mu_{q} \in \mathbb{R}$ such that

$$
-\Delta v_{q}+V(x) v_{q}=a v_{q}^{q+1}+\mu_{q} v_{q}
$$

and $v_{q}$ satisfies the Pohozaev identity in Lemma 2.3, we deduce from (3.24) and Proposition 4.1 that

$$
\frac{q-2}{2} \int_{\mathbb{R}^{2}}\left|\nabla v_{q}\right|^{2} d x+\frac{1}{2} \int_{\mathbb{R}^{2}}(q V+x \cdot \nabla V) v_{q}^{2} d x=\frac{q-2}{2} \tau_{q}^{2}+o(1)
$$

as $q \rightarrow 2_{+}$. The upper bound in (4.37) is then obtained by the assumption (V2).

The lower bound in (4.37) is obtained indirectly. Indeed, were it not true, there would exist $\left\{q_{k}\right\}$ with $q_{k} \rightarrow 2_{+}$as $k \rightarrow \infty$ such that

$$
\int_{\mathbb{R}^{2}}\left|\nabla v_{q_{k}}\right|^{2} d x=o\left(\left(q_{k}-2\right) \tau_{q_{k}}^{2}\right)
$$

as $k \rightarrow \infty$. By (4.39) and (4.40), we have

$$
\int_{\mathbb{R}^{2}}\left(q_{k} V+x \cdot \nabla V\right) v_{q_{k}}^{2} d x=O\left(\left(q_{k}-2\right) \tau_{q_{k}}^{2}\right) .
$$

The fact

$$
\frac{p}{2} V-C_{1} \leq x \cdot \nabla V \leq 2 p V+C_{2},
$$

which can be verified as (3.53), implies

$$
\frac{p}{2} \int_{\mathbb{R}^{2}} V v_{q_{k}}^{2} d x-C_{1} \leq \int_{\mathbb{R}^{2}} x \cdot \nabla V v_{q_{k}}^{2} d x \leq 2 p \int_{\mathbb{R}^{2}} V v_{q_{k}}^{2} d x+C_{2} .
$$

Therefore,

$$
\int_{\mathbb{R}^{2}} V v_{q_{k}}^{2} d x=O\left(\left(q_{k}-2\right) \tau_{q_{k}}^{2}\right), \quad \int_{\mathbb{R}^{2}} x \cdot \nabla V v_{q_{k}}^{2} d x=O\left(\left(q_{k}-2\right) \tau_{q_{k}}^{2}\right) .
$$

Hence, the Pohozaev identity (2.19) yields that

$$
\int_{\mathbb{R}^{2}}\left|\nabla v_{q_{k}}\right|^{2} d x \geq \frac{1}{2} \int_{\mathbb{R}^{2}} x \cdot \nabla V v_{q_{k}}^{2} d x=O\left(\left(q_{k}-2\right) \tau_{q_{k}}^{2}\right),
$$

which is a contradiction to (4.40). 


\section{BLOW-UP ANALYSIS}

In this section, using the blow-up argument, we study the asymptotic behavior of $v_{q}$ as $q \rightarrow 2_{+}$. This is carried through in the proof of Theorem 1.3.

Proof of Theorem 1.3. By Proposition 4.2, we have either the case $(i)$

$$
\liminf _{q \rightarrow 2_{+}} \frac{\int_{\mathbb{R}^{2}}\left|\nabla v_{q}\right|^{2} d x}{(q-2) \tau_{q}^{2}}=C>0,
$$

or the case $(i i)$

$$
\liminf _{q \rightarrow 2_{+}} \frac{\int_{\mathbb{R}^{2}}\left|\nabla v_{q}\right|^{2} d x}{(q-2) \tau_{q}^{2}}=+\infty
$$

We will treat these two cases separately.

In the case $(i)$, there exists $\left\{q_{k}\right\}$ with $q_{k} \rightarrow 2_{+}$as $k \rightarrow \infty$ such that

$$
\int_{\mathbb{R}^{2}}\left|\nabla v_{q_{k}}\right|^{2} d x=O\left(\lambda_{q_{k}}\right)
$$

where and in the following we denote $\lambda_{q_{k}}=\left(q_{k}-2\right) \tau_{q_{k}}^{2}$. We note $\lambda_{q_{k}} \rightarrow \infty$, as $k \rightarrow \infty$. Therefore, we deduce from (4.39), (4.41) and (5.1) that

$$
\int_{\mathbb{R}^{2}} V v_{q_{k}}^{2} d x=O\left(\lambda_{q_{k}}\right)
$$

and

$$
\int_{\mathbb{R}^{2}} x \cdot \nabla V v_{q_{k}}^{2} d x=O\left(\lambda_{q_{k}}\right)
$$

By (3.50) and (4.38), we have

$$
\mu_{q_{k}}=\int_{\mathbb{R}^{2}}\left|\nabla v_{q_{k}}\right|^{2} d x+\int_{\mathbb{R}^{2}} V(x) v_{q_{k}}^{2} d x-a \int_{\mathbb{R}^{2}}\left|v_{q_{k}}\right|^{q+2} d x<\lambda_{1} .
$$

Hence, by (5.4) and (5.6),

$$
a \int_{\mathbb{R}^{2}}\left|v_{q_{k}}\right|^{q+2} d x \geq \int_{\mathbb{R}^{2}} V(x) v_{q_{k}}^{2} d x-\lambda_{1}=O\left(\lambda_{q_{k}}\right) .
$$

On the other hand, by the Pohozaev identity (2.19), we have

$$
\int_{\mathbb{R}^{2}}\left|\nabla v_{q_{k}}\right|^{2} d x-\frac{1}{2} \int_{\mathbb{R}^{2}} x \cdot \nabla V\left|v_{q_{k}}\right|^{2} d x=\frac{a q_{k}}{q_{k}+2} \int_{\mathbb{R}^{2}}\left|v_{q_{k}}\right|^{q_{k}+2} d x .
$$

Thus, we obtain from (5.5) for $k$ large enough that

$$
\frac{q_{k} a}{q_{k}+2} \int_{\mathbb{R}^{2}}\left|v_{q_{k}}\right|^{q+2} d x \leq \int_{\mathbb{R}^{2}}\left|\nabla v_{q_{k}}\right|^{2} d x=O\left(\lambda_{q_{k}}\right) .
$$

Consequently,

$$
a \int_{\mathbb{R}^{2}}\left|v_{q_{k}}\right|^{q+2} d x=O\left(\lambda_{q_{k}}\right)
$$


By (5.3), there exist $C_{1}>0$ and $C_{2}>0$ such that

$$
C_{1}\left(q_{k}-2\right)\left(\frac{2 a_{q_{k}}^{*}}{q_{k} a}\right)^{\frac{2}{q_{k}-2}} \leq \int_{\mathbb{R}^{2}}\left|\nabla v_{q_{k}}\right|^{2} d x \leq C_{2}\left(q_{k}-2\right)\left(\frac{2 a_{q_{k}}^{*}}{q_{k} a}\right)^{\frac{2}{q_{k}-2}} .
$$

Let

and

$$
\varepsilon_{q}=\left\|\nabla v_{q}\right\|_{2}^{-1}
$$

Then,

$$
\tilde{w}_{q}(x)=\varepsilon_{q} v_{q}\left(\varepsilon_{q} x\right)
$$

$$
\left\|\nabla \tilde{w}_{q}\right\|_{2}^{2}=\left\|\tilde{w}_{q}\right\|_{2}^{2}=1 \text {. }
$$

It is known from Lemma 2.1 that $a_{q}^{*} \rightarrow a^{*}$ as $q \rightarrow 2_{+}$, then by (5.9),

$$
\varepsilon_{q_{k}}^{q_{k}-2}=\left(\int_{\mathbb{R}^{2}}\left|\nabla v_{q_{k}}\right|^{2} d x\right)^{\frac{2-q_{k}}{2}} \rightarrow \frac{a}{a^{*}} .
$$

Moreover, by (5.3), (5.8), (5.10) and (5.13), there exist $C_{3}>0$ and $C_{4}>0$ such that

$$
C_{3} \leq \int_{\mathbb{R}^{2}}\left|\tilde{w}_{q_{k}}\right|^{q_{k}+2} d x=\varepsilon_{q_{k}}^{q_{k}-2} \frac{\int_{\mathbb{R}^{2}}\left|v_{q_{k}}\right| q_{k}+2 d x}{\int_{\mathbb{R}^{2}}\left|\nabla v_{q_{k}}\right|^{2} d x} \leq C_{4} .
$$

Arguing as (3.41), we find from (5.12) and (5.14) that there exist $\left\{y_{q_{k}}\right\} \subset \mathbb{R}^{2}, R_{0}>0$ and $\eta>0$ such that

$$
\liminf _{k \rightarrow \infty} \int_{B_{R_{0}}\left(y_{q_{k}}\right)}\left|\tilde{w}_{q_{k}}\right|^{2} d x \geq \eta
$$

Denote $w_{q_{k}}=\tilde{w}_{q_{k}}\left(x+y_{q_{k}}\right)=\varepsilon_{q_{k}} v_{q_{k}}\left(\varepsilon_{q_{k}}\left(x+y_{q_{k}}\right)\right)$. We have

$$
\begin{aligned}
& \left\|\nabla w_{q_{k}}\right\|_{2}^{2}=\left\|w_{q_{k}}\right\|_{2}^{2}=1 ; \\
& C_{3} \leq \int_{\mathbb{R}^{2}}\left|w_{q_{k}}\right|{ }^{q_{k}+2} d x \leq C_{4} ; \\
& \liminf _{k \rightarrow \infty} \int_{B_{R_{0}}(0)}\left|w_{q_{k}}\right|^{2} d x \geq \eta .
\end{aligned}
$$

Hence, there exist a sequence $\left\{q_{k}\right\}$ with $q_{k} \rightarrow 2_{+}$as $k \rightarrow \infty$ and $w \in H^{1}\left(\mathbb{R}^{2}\right)$ such that $w_{q_{k}} \rightarrow w$ weakly in $H^{1}\left(\mathbb{R}^{2}\right)$ and $w_{q_{k}} \rightarrow w \neq 0$ strongly in $L_{l o c}^{\gamma}\left(\mathbb{R}^{2}\right)$ for any $\gamma \geq 2$. By (4.38), we see that $w_{q_{k}}$ solves

$$
-\Delta w_{q_{k}}+\varepsilon_{q_{k}}^{2} V\left(\varepsilon_{q_{k}}\left(x+y_{q_{k}}\right)\right) w_{q_{k}}=\varepsilon_{q_{k}}^{2} \mu_{q_{k}} w_{q_{k}}+\varepsilon_{q_{k}}^{2-q} a w_{q_{k}}^{q_{k}+1} .
$$

Now, we study the asymptotic behavior of $w_{q_{k}}$ and the limiting equation of (5.17).

Let us consider the limiting behavior of $\varepsilon_{q_{k}}^{2} \mu_{q_{k}}$ first. By (5.6), we have

$$
\varepsilon_{q_{k}}^{2} \mu_{q_{k}} \leq \varepsilon_{q_{k}}^{2} \lambda_{1} \rightarrow 0, \text { as } q \rightarrow 2 .
$$

On the other hand, equations (5.3), (5.6), (5.8) and (5.10) yield

$$
\varepsilon_{q_{k}}^{2} \mu_{q_{k}} \geq-\varepsilon_{q_{k}}^{2} a \int_{\mathbb{R}^{2}}\left|v_{q_{k}}\right|^{q_{k}+2} d x \geq-C \varepsilon_{q_{k}}^{2} \lambda_{q_{k}} \geq-C .
$$

So we may assume

$$
\varepsilon_{q_{k}}^{2} \mu_{q_{k}} \rightarrow-\beta^{2} \leq 0
$$


Next, we study the limiting behavior of $\varepsilon_{q_{k}}^{2} V\left(\varepsilon_{q_{k}}\left(x+y_{q_{k}}\right)\right)$.

If $\liminf _{k \rightarrow \infty}\left|\varepsilon_{q_{k}} y_{q_{k}}\right| \leq C$ with $C>0$ independent of $k$, then there exists $\left\{q_{k}\right\}$ such that $\left|\varepsilon_{q_{k}} y_{q_{k}}\right| \leq C$. So for any $M>0$, we have

$$
\varepsilon_{q_{k}}^{2} V\left(\varepsilon_{q_{k}}\left(x+y_{q_{k}}\right)\right) \leq \varepsilon_{q_{k}}^{2}\left(\left|\varepsilon_{q_{k}} x\right|+\left|\varepsilon_{q_{k}} y_{q_{k}}\right|+\max _{i}\left|x_{i}\right|\right)^{p} \rightarrow 0
$$

uniformly for $x \in B_{M}(0)$ as $k \rightarrow \infty$.

On the other hand, if $\liminf _{k \rightarrow \infty}\left|\varepsilon_{q_{k}} y_{q_{k}}\right|=+\infty$, we may find a sequence $\left\{q_{k}\right\}$ with $q_{k} \rightarrow 2_{+}$as $k \rightarrow \infty$ such that $\left|\varepsilon_{q_{k}} y_{q_{k}}\right| \rightarrow+\infty$, as $k \rightarrow \infty$. For any $M>0$, if $|x| \leq M$ and $k$ is large enough, we obtain

$$
V\left(\varepsilon_{q_{k}}\left(x+y_{q_{k}}\right)\right)=\prod_{i=1}^{n}\left|\varepsilon_{q_{k}} x-x_{i}+\varepsilon_{q_{k}} y_{q_{k}}\right|^{p_{i}} \geq \frac{1}{2^{p}} \prod_{i=1}^{n}\left|-x_{i}+\varepsilon_{q_{k}} y_{q_{k}}\right|^{p_{i}} .
$$

By (5.4),

$$
\begin{aligned}
\int_{B_{R_{0}}(0)} V\left(\varepsilon_{q_{k}}\left(x+y_{q_{k}}\right)\right) w_{q_{k}}^{2}(x) d x & \leq \int_{\mathbb{R}^{2}} V\left(\varepsilon_{q_{k}}\left(x+y_{q_{k}}\right)\right) w_{q_{k}}^{2}(x) d x \\
& =\int_{\mathbb{R}^{2}} V(x) v_{q_{k}}^{2}(x) d x \\
& \leq C \lambda_{q_{k}}
\end{aligned}
$$

Hence, for $k$ large enough,

$$
\int_{B_{R_{0}}(0)} \lambda_{q_{k}}^{-1} \prod_{i=1}^{n}\left|-x_{i}+\varepsilon_{q_{k}} y_{q_{k}}\right|^{p_{i}} w_{q_{k}}^{2}(x) d x \leq C .
$$

By $(5.15)$,

$$
\lambda_{q_{k}}^{-1} \prod_{i=1}^{n}\left|-x_{i}+\varepsilon_{q_{k}} y_{q_{k}}\right|^{p_{i}} \leq \frac{C}{\eta}
$$

Note that $\varepsilon_{q_{k}}^{-2}=O\left(\lambda_{q_{k}}\right)$ via (5.3) and (5.10), we have up to a subsequence that

$$
\varepsilon_{q_{k}}^{2} \prod_{i=1}^{n}\left|-x_{i}+\varepsilon_{q_{k}} y_{q_{k}}\right|^{p_{i}} \rightarrow \mu \geq 0
$$

It is readily to verify that for any $M>0$ and $x \in B_{M}(0)$,

$$
\lim _{k \rightarrow \infty} \varepsilon_{q_{k}}^{2} V\left(\varepsilon_{q_{k}}\left(x+y_{q_{k}}\right)\right)=\lim _{k \rightarrow \infty} \varepsilon_{q_{k}}^{2} \prod_{i=1}^{n}\left|-x_{i}+\varepsilon_{q_{k}} y_{q_{k}}\right|^{p_{i}} .
$$

Hence, (5.22) implies that either

$$
\varepsilon_{q_{k}}^{2} V\left(\varepsilon_{q_{k}}\left(x+y_{q_{k}}\right)\right) \rightarrow \mu>0
$$

or

$$
\varepsilon_{q_{k}}^{2} V\left(\varepsilon_{q_{k}}\left(x+y_{q_{k}}\right)\right) \rightarrow 0
$$

uniformly in $x \in B_{M}(0)$.

In summary of $(5.18),(5.19),(5.23)$ and $(5.24)$, in the case $(i)$ we have the following subcases: 
Subcase $(i): \quad \varepsilon_{q_{k}}^{2} \mu_{q_{k}} \rightarrow 0$, and (5.23) holds;

Subcase (ii): $\varepsilon_{q_{k}}^{2} \mu_{q_{k}} \rightarrow 0$, and (5.24) holds;

Subcase (iii): $\varepsilon_{q_{k}}^{2} \mu_{q_{k}} \rightarrow-\beta^{2}<0$, and (5.23) holds;

Subcase (iv): $\varepsilon_{q_{k}}^{2} \mu_{q_{k}} \rightarrow-\beta^{2}<0$, and (5.24) holds.

Taking the limit $k \rightarrow \infty$ in (5.17), we obtain that $w$ satisfies correspondingly in the subcase $(i)$ that

$$
-\Delta w+\mu w=a^{*} w^{3}
$$

in the subcase $(i i)$ that

$$
-\Delta w=a^{*} w^{3}
$$

in the subcase $($ iii) that

$$
-\Delta w+\left(\mu+\beta^{2}\right) w=a^{*} w^{3}
$$

and in the subcase $(i v)$ that

$$
-\Delta w+\beta^{2} w=a^{*} w^{3} .
$$

In the subcase $(i)$, by the uniqueness of positive solution of $(5.25)$ and $w \neq 0$, there exists $y_{0} \in \mathbb{R}^{2}$ such that

$$
w=\frac{\sqrt{\mu}}{\|Q\|_{2}} Q\left(\sqrt{\mu}\left(x-y_{0}\right)\right),
$$

which implies $\|w\|_{2}^{2}=1$. Hence, $w_{q_{k}} \rightarrow w$ strongly in $L^{2}\left(\mathbb{R}^{2}\right)$, that is

$$
\varepsilon_{q_{k}} v_{q_{k}}\left(\varepsilon_{q_{k}}\left(x+y_{q_{k}}\right)\right) \rightarrow \frac{\sqrt{\mu}}{\|Q\|_{2}} Q\left(\sqrt{\mu}\left(x-y_{0}\right)\right) \text { strongly in } L^{2}\left(\mathbb{R}^{2}\right) .
$$

The subcase $(i i)$ can not happen. Indeed, if it would happen on the contrary, the fact that $w \geq 0$ and $w \neq 0$ would imply that (5.26) admits a positive solution, which contradicts the Liouville type theorem.

In the same way to drive (5.29), we can show in the subcase (iii) that, there exists $y_{1} \in \mathbb{R}^{2}$ such that

$$
\varepsilon_{q_{k}} v_{q_{k}}\left(\varepsilon_{q_{k}}\left(x+y_{q_{k}}\right)\right) \rightarrow \frac{\sqrt{\mu+\beta^{2}}}{\|Q\|_{2}} Q\left(\sqrt{\mu+\beta^{2}}\left(x-y_{1}\right)\right) \text { strongly in } L^{2}\left(\mathbb{R}^{2}\right),
$$

and in the subcase $(i v)$ that, there exists $y_{2} \in \mathbb{R}^{2}$ such that

$$
\varepsilon_{q_{k}} v_{q_{k}}\left(\varepsilon_{q_{k}}\left(x+y_{q_{k}}\right)\right) \rightarrow \frac{\beta}{\|Q\|_{2}} Q\left(\beta\left(x-y_{2}\right)\right) \text { strongly in } L^{2}\left(\mathbb{R}^{2}\right) .
$$

Therefore, the conclusions in Theorem 1.3 are valid for the case $(i)$.

Now, we turn to the case $(i i)$.

By (5.2), there exists $\left\{q_{k}\right\}$ such that

$$
\int_{\mathbb{R}^{2}}\left|\nabla v_{q_{k}}\right|^{2} d x>>O\left(\lambda_{q_{k}}\right)
$$

Then, equation (4.39) and (4.41) imply

$$
\int_{\mathbb{R}^{2}} V v_{q_{k}}^{2} d x \leq C \lambda_{q_{k}},
$$


which yields via (4.41) that

$$
\left|\int_{\mathbb{R}^{2}} x \cdot \nabla V v_{q_{k}}^{2} d x\right| \leq C \lambda_{q_{k}}
$$

By the Pohozaev identity (5.7) and (4.41),

$$
\lim _{k \rightarrow \infty} \frac{\int_{\mathbb{R}^{2}}\left|\nabla v_{q_{k}}\right|^{2} d x}{\frac{a}{2} \int_{\mathbb{R}^{2}}\left|v_{q_{k}}\right|^{q_{k}+2} d x}=1
$$

Now, we apply the blow-up analysis for $\tilde{w}_{q_{k}}$, which is defined in (5.11).

By (4.37), (5.10) and (5.35),

$$
\varepsilon_{q_{k}}^{q_{k}-2}=\left(\int_{\mathbb{R}^{2}}\left|\nabla v_{q_{k}}\right|^{2} d x\right)^{\frac{2-q}{2}} \geq\left(2 \tau_{q_{k}}^{2}\right)^{\frac{q-2}{2}}=2^{\frac{q-2}{2}} \frac{q a}{2 a_{q}^{*}} \rightarrow \frac{a}{a^{*}}
$$

and

$$
\varepsilon_{q_{k}}^{q_{k}-2} \leq\left(C \lambda_{q_{k}}\right)^{\frac{2-q}{2}}=C^{\frac{q-2}{2}}(q-2)^{\frac{q-2}{2}} \frac{q a}{2 a_{q}^{*}} \rightarrow \frac{a}{a^{*}}
$$

Thus, we have

$$
\varepsilon_{q_{k}}^{q_{k}-2} \rightarrow \frac{a}{a^{*}}
$$

By (5.35), there exist $C_{1}>0$ and $C_{2}>0$ such that

$$
C_{1} \leq \int_{\mathbb{R}^{2}}\left|\tilde{w}_{q_{k}}\right|^{q_{k}+2} d x=\varepsilon_{q_{k}}^{q_{k}-2} \frac{\left.\int_{\mathbb{R}^{2}}\left|v_{q_{k}}\right|\right|^{q_{k}+2} d x}{\int_{\mathbb{R}^{2}}\left|\nabla v_{q_{k}}\right|^{2} d x} \leq C_{2} .
$$

Proceeding as the case $(i)$, there exists $y_{q_{k}} \in \mathbb{R}^{2}$ such that (5.15) holds. Let

$$
w_{q_{k}}=\tilde{w}_{q_{k}}\left(x+y_{q_{k}}\right)=\varepsilon_{q_{k}} v_{q}\left(\varepsilon_{q_{k}}\left(x+y_{q_{k}}\right)\right) .
$$

Hence, there is a subsequent of $\left\{w_{q_{k}}\right\}$, still denoted by $\left\{w_{q_{k}}\right\}$, such that $w_{q_{k}} \rightarrow w \neq 0$ weakly in $H^{1}\left(\mathbb{R}^{2}\right)$ and $w_{q_{k}} \rightarrow w$ strongly in $L_{l o c}^{\gamma}\left(\mathbb{R}^{2}\right)$ for any $\gamma \geq 2$. Moreover, $w_{q}$ solves (5.17).

In the same way as the case $(i)$, we analyze the limiting behavior of $\varepsilon_{q_{k}}^{2} V\left(\varepsilon_{q_{k}}\left(x+y_{q_{k}}\right)\right)$. If $\liminf _{k \rightarrow \infty}\left|\varepsilon_{q_{k}} y_{q_{k}}\right| \leq C$ with $C>0$ independent of $k$, there exists $\left\{q_{k}\right\}$ such that $\left|\varepsilon_{q_{k}} y_{q_{k}}\right| \leq C$ and (5.23) holds.

If $\liminf _{k \rightarrow \infty}\left|\varepsilon_{q_{k}} y_{q_{k}}\right|=\infty$, then there exists $\left\{q_{k}\right\}$ such that $\left|\varepsilon_{q_{k}} y_{q_{k}}\right| \rightarrow \infty$. By (5.33), we proceed as the case $(i)$ that (5.21) also holds. Hence, for any $x \in B_{M}(0)$, due to (5.10) and 
(5.32), we find

$$
\begin{aligned}
\varepsilon_{q_{k}}^{2} V\left(\varepsilon_{q_{k}}\left(x+y_{q_{k}}\right)\right) & \leq \varepsilon_{q_{k}}^{2} \prod_{i=1}^{n}\left(\left|\varepsilon_{q_{k}} x\right|+\left|-x_{i}+\varepsilon_{q_{k}} y_{q_{k}}\right|\right)^{p_{i}} \\
& \leq 2 \varepsilon_{q_{k}}^{2} \prod_{i=1}^{n}\left|-x_{i}+\varepsilon_{q_{k}} y_{q_{k}}\right|^{p_{i}} \\
& \leq 2 \varepsilon_{q_{k}}^{2} \lambda_{q_{k}} \lambda_{q_{k}}^{-1} \prod_{i=1}^{n}\left|-x_{i}+\varepsilon_{q_{k}} y_{q_{k}}\right|^{p_{i}} \\
& \leq \frac{C \varepsilon_{q_{k}}^{2} \lambda_{q_{k}}}{\eta} \rightarrow 0 .
\end{aligned}
$$

Now, we treat the factor $\varepsilon_{q_{k}}^{2} \mu_{q_{k}}$. By (5.6), (5.35), (5.32) and (5.33), we have $-\mu_{q_{k}}=$ $O\left(\varepsilon_{q_{k}}^{-2}\right)$. Hence, there exists $\left\{q_{k}\right\}$ and $\beta>0$ such that $\varepsilon_{q_{k}}^{2} \mu_{q_{k}} \rightarrow-\beta^{2}$. Taking the limit in (5.17), we see that $w$ satisfies

$$
-\Delta w+\beta^{2} w=a^{*} w^{3} .
$$

Similar to the proof in the subcase $(i)$, there exists $y_{0} \in \mathbb{R}^{2}$ and $\left\{v_{q_{k}}\right\}$ such that

$$
\varepsilon_{q_{k}} v_{q_{k}}\left(\varepsilon_{q_{k}}\left(x+y_{q_{k}}\right)\right) \rightarrow \frac{\beta}{\|Q\|_{2}} Q\left(\beta\left(x-y_{0}\right)\right) \text { strongly in } L^{2}\left(\mathbb{R}^{2}\right) .
$$

Equation (1.13) then follows from (5.29)-(5.31) and (5.38). The proof is complete.

Finally, we deal with the special case: $V(x)=|x|^{p}$ with $p \geq 1$.

Proof of Corollary 1.2. In the case $V(x)=|x|^{p}, v_{q}$ satisfies

$$
-\Delta v_{q}+|x|^{p} v_{q}=\mu_{q} v_{q}+v_{q}^{q+1} \text {. }
$$

By the classical bootstrap argument, we have $v_{q} \in C^{2}\left(\mathbb{R}^{2}\right)$ and $\lim _{|x| \rightarrow \infty} v_{q}(x)=0$. We know from Theorem 2 in [16] that $v_{q}$ is radially symmetric and decreasing from the origin. Since the Sobolev inclusion $H_{r}^{1}\left(\mathbb{R}^{2}\right) \hookrightarrow L^{\gamma}\left(\mathbb{R}^{2}\right)$ is compact for any $\gamma>2$, we may choose $y_{q}=0$ in (5.15), and it is readily to show that (5.24) holds true.

We may verify through the proof of Theorem 1.3 that only subcase $(i v)$ and case $(i i)$ may happen, that is,

$$
\varepsilon_{q_{k}}^{2} \mu_{q_{k}} \rightarrow-\beta^{2}<0
$$

and

$$
\varepsilon_{q_{k}}^{2-q} a \rightarrow a^{*}
$$

By (5.17), we have

$$
-\Delta w_{q_{k}} \leq\left(\varepsilon_{q}^{2-q} a w_{q_{k}}^{q_{k}}\right) w_{q_{k}} .
$$

Using the De Diorgi-Nash-Moser estimate, see Theorem 4.1 in [9], we obtain

$$
\max _{B_{1}(\xi)} w_{q_{k}} \leq C\left(\int_{B_{2}(\xi)}\left|w_{q_{k}}\right|^{\gamma} d x\right)^{\frac{1}{\gamma}}
$$


for any $\gamma \geq 2$ and $\xi \in \mathbb{R}^{2}$. The constant $C$ depends only on the bound of $\left\|w_{q_{k}}\right\|_{L^{\gamma}\left(B_{2}(\xi)\right)}$. It follows from $\left\|w_{q}\right\|_{H^{1}\left(\mathbb{R}^{2}\right)}^{2}=1, H^{1}\left(\mathbb{R}^{2}\right) \hookrightarrow L^{\gamma}\left(\mathbb{R}^{2}\right)$ for any $\gamma \geq 2$ and (5.40) that $w_{q_{k}}$ is uniformly bounded in $L^{\infty}\left(\mathbb{R}^{2}\right)$.

By Lemma 1.7.3 in [6] and $\left\|w_{q_{k}}\right\|_{2}^{2}=1$,

$$
w_{q}(x) \leq \frac{C}{|x|} .
$$

So there exists $R>0$, independent of $k$, and $k$ large enough, for $|x| \geq R$ that,

$$
-\Delta w_{q_{k}} \leq-\frac{1}{3} \beta^{2} w_{q_{k}} .
$$

By the comparison principle,

$$
w_{q_{k}}(x) \leq C e^{-\frac{1}{4} \beta^{2}|x|}
$$

for $|x| \geq R$. Since $w_{q_{k}}$ is uniformly bounded in $L^{\infty}\left(\mathbb{R}^{2}\right)$, we have

$$
w_{q_{k}}(x) \leq C e^{-\frac{1}{4} \beta^{2}|x|} .
$$

for any $x \in \mathbb{R}^{2}$, where $C$ is independent of $k$. Therefore,

$$
\int_{\mathbb{R}^{2}} V(x) v_{q_{k}}^{2} d x=\int_{\mathbb{R}^{2}} V\left(\varepsilon_{q_{k}} x\right) w_{q_{k}}^{2} d x \leq C \int_{\mathbb{R}^{2}} \varepsilon_{q_{k}}^{p}|x|^{p} e^{-\frac{1}{2} \beta^{2}|x|} d x \rightarrow 0 .
$$

The inequalities (4.41) and (5.42) yield

$$
\left|\int_{\mathbb{R}^{2}} x \cdot \nabla V v_{q_{k}}^{2} d x\right| \leq C
$$

We conclude from (4.39), (5.42) and (5.43) that

$$
\lim _{k \rightarrow \infty} \tau_{q_{k}}^{-2} \int_{\mathbb{R}^{2}}\left|\nabla v_{q_{k}}\right|^{2} d x=1,
$$

and case $(i)$ can not happen.

By (5.7), (5.43) and (5.44), we obtain

$$
\lim _{k \rightarrow \infty} \frac{\int_{\mathbb{R}^{2}}\left|\nabla v_{q_{k}}\right|^{2} d x}{\frac{a}{2} \int_{\mathbb{R}^{2}}\left|v_{q_{k}}\right|^{q_{k}+2} d x}=1 .
$$

It follows from (5.6) and (5.42)-(5.45) that $\lim _{q_{k} \rightarrow \infty} \frac{\mu_{q_{k}}}{\tau_{q_{k}}^{2}}=-1$, and then $\varepsilon_{q_{k}}^{2} \mu_{q_{k}} \rightarrow-1$. By (5.18), $\beta^{2}=1$. The proof is complete.

Acknowledgment The first author is supported by NNSF of China, No:11671179 and 11771300. The second author is supported by NNSF of China, No:11701260. 


\section{REFERENCES}

[1] A. Azzollini and A. Pomponio, On the Schrödinger equation in $R^{N}$ under the effect of a general nonlinear term, Indiana Univ. Math. J., 58 (2009), no. 3, 1361-1378.

[2] T. Bartsch and N. Soave, A natural constraint approach to normalized solutions of nonlinear Schröinger equations and systems, Journal of Functional Analysis, 272(2017), 4998-5037.

[3] T. Bartsch, S. de Valeriola, Normalized solutions of nonlinear Schröinger equations, Arch. Math., 100(1) (2012), 75-83.

[4] J. Bellazzini, L. Jeanjean, On dipolar quantum gases in the unstable regime, SIAM J. Math. Anal., 48,(2016), 2028-2058.

[5] H. Berestycki and P. L. Lions, Nonlinear scalar field equations, I and II, Arch. Rat. Mech. Anal., 82 (1983), 313-346, 347-375.

[6] T. Cazenave, Semilinear Schrodinger Equations, Courant Lecture Notes in Mathematics Vol. 10, Courant Institute of Mathematical Science/AMS New York, 2003.

[7] F. Dalfovo, S. Giorgini, L.P. Pitaevskii, S. Stringari, Theory of Bose-Einstein condensation in trapped gases, Rev. Mod. Phys., 71,(1999), 463-512.

[8] N. Ghoussoub, Duality and Perturbation Methods in Critical Point Theory, Cambridge Tracts in Mathematics, vol. 107, Cambridge University Press, Cambridge, 1993, with appendices by David Robinson.

[9] Q. Han and F. H. Lin, Elliptic Partial Differential Equations: Second Edition, Courant Lecture Notes in Mathematics Vol. 1, Courant Institute of Mathematical Science/AMS, New York, 2011.

[10] C. Huepe, S. Metens, G. Dewel, P. Borckmans, M.E. Brachet, Decay rates in attractive Bose-Einstein condensates. Phys. Rev. Lett., 82, (1999), 1616-1619.

[11] Y. J. Guo and R. Seiringer, On the mass concentration for Bose-Einstein condensates with attractive interactions, Lett. Math. Phys. 104, (2014), 141-156.

[12] Y. J. Guo, X. Y. Zeng and H. S. Zhou, Concentration behavior of standing waves for almost mass critical nonlinear Schrödinger equations, J. Differential Equations, 256 (2014), 2079-2100.

[13] L. Jeanjean. Existence of solutions with prescribed norm for semilinear elliptic equations. Nonlinear Anal., 28(10) (1997), 1633-1659.

[14] Y. Kagan, A.E. Muryshev, G.V. Shlyapnikov, Collapse and Bose-Einstein condensation in a trapped Bose gas with negative scattering length. Phys. Rev. Lett., 81, (1998), 933-937.

[15] M.K. Kwong, Uniqueness of positive solutions of $\Delta u-u+u^{p}=0$ in $\mathbb{R}^{N}$, Arch. Ration. Mech. Anal., 105,(1989), 243-266.

[16] Y. Li and W.M. Ni, Radial symmetry of positive solutions of nonlinear elliptic equations in $\mathbb{R}^{n}$, Comm. Partial Differential Equations 18 (1993), 1043-1054.

[17] M. I. Weinstein, Nonlinear Schrödinger equations and sharp interpolation estimates, Comm. Math. Phys., 87,(1983), 567-576.

[18] Jianfu Yang and Jinge Yang, Existence and mass concentration of pseudo-relativistic Hartree equation, Journal of Mathematical Physics 58, 081501 (2017); doi: 10.1063/1.4996576.

[19] C.A. Sackett, H.T.C. Stoof, R.G. Hulet, Growth and collapse of a Bose-Einstein condensate with attractive interactions. Phys. Rev. Lett., 80,(1998), 20-31.

[20] M. Willem, Minimax Theorems, Birkhauser, Boston, Basel, Berlin, 1996. 